\title{
Health-Related Quality of Life and Oropharyngeal Dysphagia: A Systematic Review
}

Ellie Jones ${ }^{1}$, Renée Speyer ${ }^{1,2,3,6}$, Berit Kertscher ${ }^{4}$, Deborah Denman ${ }^{3}$, Katina Swan ${ }^{3,5}$, Reinie Cordier $^{3}$

${ }^{1}$ School of Public Health, Tropical Medicine and Rehabilitation Sciences, James Cook University, Townsville, Queensland, Australia

${ }^{2}$ Department of Otorhinolaryngology and Head and Neck Surgery, Leiden University Medical Center, Leiden, the Netherlands

${ }^{3}$ School of Occupational Therapy and Social Work, Curtin University, Perth, Western Australia

${ }^{4}$ RehaA Winterthur, RehaClinic, Zurzach, Switzerland

${ }^{5}$ Gold Coast Health Service, Queensland Health, Australia

${ }^{6}$ Faculty of Educational Sciences, University of Oslo, Oslo, Norway

Corresponding author:

Dr. Renée Speyer, Discipline of Speech Pathology, James Cook University, QLD, Australia, 4811. E-mail: renee.speyer@jcu.edu.au. Facsimile: +61 74781 6868. Telephone: +61 74781 6677. 


\title{
Health-Related Quality of Life and Oropharyngeal Dysphagia: A Systematic Review
}

\author{
Abstract \\ Purpose \\ The negative impact of increased bolus modification on health-related quality of life \\ (HRQoL) in persons with oropharyngeal dysphagia (OD) has been described in previous \\ literature; however, findings may have been influenced by the severity of OD and underlying \\ etiology. The current systematic review therefore aims to investigate the relationships \\ between HRQoL and OD and to report on changes in HRQoL and OD following \\ intervention.

\section{Methods}

This review was conducted in accordance with the PRISMA statement. A systematic search of the literature was performed using PubMed and Embase. All available English publications up to February 2016 that reported on OD and HRQoL were included. Articles that studied OD arising from esophageal dysfunction or included less than 15 participants with OD, were excluded.

Results

Thirty-five studies met the inclusion criteria. Inverse bidirectional relationships were found between decreased HRQoL and increased OD severity. Following intervention, changes were evident through improved HRQoL with decreased OD severity.

\section{Conclusion}

The findings of this paper highlight the importance of targeting HRQoL in patients with OD. However, because of the heterogeneity in methodology, terminology and assessment procedures used in the retrieved articles, the generalization of study results is limited. More research investigating the relationships between HRQoL and OD is needed. Future studies 
should implement study designs that lead to stronger levels of evidence, quantify the severity of OD and underlying diseases, use validated measures and less ambiguous terminology.

Keywords

Oropharyngeal Dysphagia, Health-Related Quality of Life, Deglutition 


\section{Introduction}

Oropharyngeal dysphagia (OD) refers to any abnormality in swallowing physiology of the upper digestive tract $[1,2]$. OD may affect components of the oral phase such as saliva management, mastication and bolus propulsion, and the pharyngeal phase including bolus passage, swallow initiation and airway protection [3, 4]. Dysphagia definitions vary between literature to encompass components of the oral and pharyngeal phase, precipitating oropharyngeal dysphagia and esophageal dysphagia [5] , or alternatively more broadly defined as swallowing difficulties [6-8]. Despite definitions of OD varying within literature, the impact of OD onto swallowing ranges from mild discomfort to complete incapacitation and reduced airway patency [9]. OD can lead to dehydration, malnutrition, and higher risk for pulmonary complications from aspiration and asphyxiation $[10,11]$. Early assessment, diagnosis and management are essential to minimize the risks of OD [12]. Accordingly, practice guidelines suggest implementing early evaluation with objective gold standard assessments such as videofluoroscopy (VFS) and fiberoptic endoscopic evaluation of swallowing (FEES) [13].

International prevalence data on OD in the general population varies between $2.3 \%$ and $16.0 \%[12]$. The risk of OD increases with age as a result of muscle atrophy and natural ageing processes, manifesting with increased aspiration risk in as many as $35 \%$ of the elderly population older than 75 years $[12,14,15]$. The prevalence of OD is higher with comorbid conditions, including head and neck cancer (HNC) and neurological diseases including Parkinson's disease (PD) and stroke $[6,16,17]$. While considering the potential impact of location, severity, type of cancer treatment and follow-up durations, the prevalence of OD in patients with $\mathrm{HNC}$ is a frequent complication $(50.6 \%)[18,19]$. OD is also a common consequence of PD, with coughing, choking episodes, and globus sensation being reported in up to $80 \%$ of patients during the early stages of $\mathrm{PD}$, and $100 \%$ of patients in the later stages 
[17]. The prevalence of OD amongst stroke populations is a commonly documented morbidity, but ranging widely between $19 \%$ and $81 \%[2,20]$.

Quality of life (QoL) refers to how individuals perceive their lives in the context of their culture and standards of living [21, 22]. QoL is defined as "a state of complete physical, mental and social well-being and not merely the absence of disease or infirmity". Speyer et al. (2011) suggest when quantifying patients' current health status, the inclusion of a well validated QoL measure specific to their health is an important consideration [23]. Health related quality of life (HRQoL), in contrast to QoL, is a broad subjective construct that determines individuals' perception of the impact of their health status onto different domains of their life $[10,23]$. HRQoL is defined as the impact that functional health status (FHS), illness and treatment have onto individuals' QoL, directly relating to physical, emotional, mental and social functioning [21, 24]. FHS refers to an individual's ability to perform daily roles and activities required to meet basic needs, and maintain their health and wellbeing [25]. As HRQoL is not the same as general QoL or FHS, it is important to explore all three different concepts with different outcome measures.

Recently, Swan et al. [9] described in a systematic review the negative impact of increased bolus modification on HRQoL in persons with OD. However, the authors underlined that these findings may have been influenced by the severity of OD and underlying etiology. Given the serious and disabling symptoms of OD and the potential negative impact it may have on individuals' HRQoL and wellbeing, the need to explore the nature of the relationship between HRQoL and OD has been touted in literature [7, 26-28]. Finizia, Rudberg [7] suggested combining instrumental evaluation measures such as VFS and FEES with patient reported outcome instruments to better reflect the dysphagic patient's perspective. Furthermore, Timmerman, Speyer [28] emphasized using HRQoL measures with sound psychometric properties only. As such there is a need to augment traditional clinician- 
driven assessment focussing on physiological parameters to examine swallowing impairment with measuring the impact of OD on HRQoL [7]; most HRQoL questionnaires are patient reported and do not necessarily correlate with clinician-driven measures or perception [29].

Investigating the relationships between HRQoL and OD will inform diagnostic assessment and individualized management from a discipline-specific and multidisciplinary point of view, to preserve the wellbeing of patients and their families [9]. Based on current literature we hypothesize that decreased HRQoL will be inversely related to increased severity of OD symptomology. Furthermore, a decrease in the severity of OD symptomology will lead to significant increases in HRQoL following intervention, and vice versa. Therefore, the purpose of this study is twofold: 1) to describe the relationships between HRQoL and OD and 2) report changes in OD and HRQoL following intervention.

\section{Methods}

Preferred Reporting Items for Systematic Reviews and Meta-Analyses [30] was the implemented methodology for this review. The PRISMA Statement [30] involves the completion of a four-phase flow diagram documenting data collection stages and a 27 -item checklist to guide reporting of the systematic review.

A systematic search of the literature was performed using two electronic medical databases: PubMed and Embase. As outlined in Table 1, subject headings (MeSH and Thesaurus) were supplemented by free text words. All available English publications up to $8^{\text {th }}$ February 2016 were included. Two researchers independently reviewed the abstracts and flagged articles for full-text retrieval if they, based on reviewer consensus, met the following inclusion criteria: 1) reported on OD, 2) examined HRQoL using at least one assessment, 3) reported on HRQoL in relation to OD, 4) written in English, and 5) full-text retrievable. Exclusion criteria were: 1) OD arising from esophageal dysfunction, 2) insufficient 
information to extract meaningful data for this study, and 3) studies including less than 15 participants with OD as these studies were considered to provide limited evidence. Reference lists of included articles were checked for further studies that may meet eligibility criteria. All study designs reporting on information related to our purpose were included.

The methodological quality of included articles was evaluated by two independent reviewers using the QualSyst critical appraisal tool by Kmet, Lee [31]. Advantages of using QualSyst include detailed and reproducible assessment of differences in scores within various study designs and across research paradigms, useful when synthesizing information and exploring the heterogeneity of study results. Included articles were additionally classified according to the Australian National Health and Medical Research Council Evidence Hierarchy [32], thus implementing an internationally accepted classification and assigning levels of evidence according to research design.

Table 1 Search strategies per database

\begin{tabular}{|c|c|}
\hline Database & earch strategies \\
\hline PubMed & $\begin{array}{l}\text { ("Deglutition Disorders"[Mesh:NoExp] OR "deglutition disorders"[All Fields] OR } \\
\text { "oropharyngeal dysphagia"[All Fields] OR "deglutition"[Mesh Terms] OR } \\
\text { "deglutition"[All Fields] OR "swallowing"[All Fields]) AND ("dysfunction"[All Fields] } \\
\text { OR "pain"[Mesh Terms] OR "pain"[All Fields] OR "problem"[All Fields] OR } \\
\text { "problems"[All Fields] OR "disease"[Mesh Terms] OR "disease"[All Fields] OR } \\
\text { "disorder"[All Fields] OR "disease"[All Fields] OR "disorders"[All Fields]) AND } \\
\text { ("Quality of Life"[Mesh] OR "Quality of Life"[all fields] OR "health related quality of } \\
\text { life"[all fields] OR HRQL[All Fields] OR HRQoL[All Fields] OR QoL[all fields]) }\end{array}$ \\
\hline Emb & $\begin{array}{l}\text { (*dysphagia/ OR "deglutition disorders".ti. OR "oropharyngeal dysphagia".ti. OR } \\
\text { *swallowing/ OR "deglutition".ti. OR "swallowing".ti.) AND ("dysfunction".ti. OR *pain/ } \\
\text { OR "pain*".ti. OR "problem*".ti. OR *diseases/ OR "disease*".ti. OR "disorder*".ti.) } \\
\text { AND (exp "quality of life"/ OR "Quality of Life".mp. OR "health related quality of } \\
\text { life".mp. OR HRQL.mp. OR HRQoL.mp. OR QoL.mp.) }\end{array}$ \\
\hline
\end{tabular}




\section{Results}

\section{Study selection}

A total of 2,118 abstracts (excluding duplicates) were yielded through literature searches within PubMed and Embase. 1,790 were immediately excluded for not meeting the eligibility criteria. Full text articles were accessed for the remaining 328 articles, 35 of which met all inclusion criteria, relating to HRQoL and OD. One article was added through reference lists of the included articles. Figure 1 outlines further detail on the PRISMA reviewing process according to Moher [30].

\section{Quality assessment}

The methodological quality of all originally accepted articles was based on the QualSyst ratings [29], ranging from poor $<50 \%$, adequate $50-59 \%$, strong $60-79 \%$, to good $>80 \%$. One article ranked as poor and was therefore excluded from the review [33], one article ranked as adequate [34], 13 articles ranked as strong, and 21 articles as good (see Table 2). NHMRC Evidence Hierarchy [32] designates the following hierarchy: Level I (evidence obtained from a systematic review of all relevant RCTs), level II (evidence obtained from at least one properly designed RCT), level III-1 (evidence obtained from well-designed pseudo-RCTs [alternate allocation or some other method]), level III-2 (evidence obtained from comparative studies with concurrent controls and allocation not randomized [cohort studies], case control studies, or interrupted time series with a control group), level III-3 (evidence obtained from comparative studies with historical control, two or more single-arm studies, or interrupted time series without a parallel control group), and level IV (evidence obtained from case series, either post-test or pre-test and post-test). Based on the above NHMRC Evidence Hierarchy four articles were classified as level III-1 evidence and 31 articles as level IV evidence. 
Table 2 Methodological quality and NHMRC evidence level of all included articles

\begin{tabular}{|c|c|c|c|}
\hline Reference & $\begin{array}{l}\text { Kmet score } \\
(\%)\end{array}$ & $\begin{array}{l}\text { Kmet Methodological } \\
\text { Quality }\end{array}$ & $\begin{array}{l}\text { NHMRC Level } \\
\text { of Evidence }\end{array}$ \\
\hline Airoldi et al., 2010 [16] & $14 / 22(63.6 \%)$ & Strong & IV \\
\hline Argolo et al., 2013 [35] & $21 / 24(87.5 \%)$ & Good & IV \\
\hline Asadollahpour et al., 2015 [36] & $20 / 22(90.9 \%)$ & Good & IV \\
\hline Bibi et al., 2015 [37] & $17 / 22(77.2 \%)$ & Strong & IV \\
\hline Bogaardt et al., 2009 [26] & $19 / 22(86.3 \%)$ & Good & IV \\
\hline Campbell et al., 2004 [38] & $19 / 24(79.1 \%)$ & Strong & IV \\
\hline Carlsson et al., 2012 [39] & $21 / 22(95.5 \%)$ & Good & IV \\
\hline $\begin{array}{l}\text { da Costa Franceschini et al., } 2015 \\
{[40]}\end{array}$ & $22 / 22(100 \%)$ & Good & IV \\
\hline da Silva Brandao et al., $2010[6]$ & $18 / 24(75 \%)$ & Strong & IV \\
\hline dos Santos Queija et al., 2009[41] & $18 / 24(75 \%)$ & Strong & IV \\
\hline Eslick and Talley, 2008 [14] & $18 / 22(81.8 \%)$ & Good & IV \\
\hline Finizia et al., 2012 [7] & $20 / 22(90.9 \%)$ & Good & IV \\
\hline Florie et al., 2015 [42] & $22 / 24(91.7 \%)$ & Good & IV \\
\hline García-Peris et al., 2007 [18] & $15 / 22(68.2 \%)$ & Strong & IV \\
\hline Heijnen et al., 2012 [43] & $22 / 26(84.6 \%)$ & Good & III-1 \\
\hline Lal et al., 2014[44] & $17 / 22(77.2 \%)$ & Strong & IV \\
\hline Lam \& Lai, 2011 [45] & $19 / 22(86.3 \%)$ & Good & IV \\
\hline Leow et al., 2010 [46] & $21 / 22(95.5 \%)$ & Good & IV \\
\hline Lin et al., 2011 [47] & $22 / 26(84.6 \%)$ & Good & III-1 \\
\hline Lovell et al., 2005 [48] & $20 / 22(90.9 \%)$ & Good & IV \\
\hline Maclean et al., 2009 [19] & $20 / 22(90.9 \%)$ & Good & IV \\
\hline Maurer et al., 2011[33] & $8 / 22(36.4 \%)$ & Poor & NA \\
\hline McHorney et al., 2002 [49] & $17 / 22(77.2 \%)$ & Strong & IV \\
\hline McHorney et al., 2006[27] & $19 / 24(79.2 \%)$ & Strong & IV \\
\hline Nguyen et al., 2005 [50] & $17 / 26(65.4 \%)$ & Strong & IV \\
\hline Nogueira et al., 2015[51] & $18 / 22(81.8 \%)$ & Good & IV \\
\hline Pedersen et al., 2016 [52] & $20 / 22(90.9 \%)$ & Good & IV \\
\hline Pierce et al., 2015 [53] & $19 / 22(86.4 \%)$ & Good & IV \\
\hline Ramaekers et al., 2011 [54] & $19 / 24(79.2 \%)$ & Strong & IV \\
\hline Rogus-Pulia et al., 2016 [55] & $19 / 24(79.2 \%)$ & Strong & IV \\
\hline Starmer et al., 2014 [56] & $19 / 22(86.4 \%)$ & Good & IV \\
\hline Tabor et al., 2016 [57] & $20 / 24(83.3 \%)$ & Good & IV \\
\hline Vanderwegen et al., 2012[58] & $20 / 22(90.9 \%)$ & Good & IV \\
\hline Zhang et al., 2014 [59] & $17 / 24(70.8 \%)$ & Strong & IV \\
\hline Zhang et al., 2016 [60] & $22 / 26(84.6 \%)$ & Good & III-1 \\
\hline Zheng et al., 2014 [34] & $12 / 22(54.5 \%)$ & Adequate & III-1 \\
\hline
\end{tabular}

Note. NA: Not Applicable

\section{Overview of included studies}

The final number of studies accepted was 35 studies published between 2002 and 2016. Data relating to HRQoL of persons with OD were extracted from the included articles, collated 
and presented descriptively. Table 3 summarizes included 27 articles reporting on the relationships between HRQoL and OD and Table 4 summarizes eight articles on changes in HRQoL and OD following intervention. The term intervention broadly refers to any intervention undertaken, including speech pathology and/ or pharmacological intervention. The first columns list all studies alphabetically by lead author. Subsequent columns outline data retrieved on participant characteristics, assessments including gold standards used (if applicable), intervention type and groups (if applicable), and key findings related to the purpose of this review.

In line with higher prevalence of OD amongst comorbid medical conditions, most included articles reported on HRQoL in HNC (15 articles) $[16,18,19,34,38,41,42,44,47$, 48, 50, 52, 54, 56, 59], neurological diseases including PD (five articles) [35, 40, 43, 46, 57], stroke (two articles) [6, 60], or a combination of the above (11 articles) [7, 26, 27, 36, 37, 39, $45,49,51,55,58]$. One article described patients with Sjogren's syndrome [53] and another article studied the general population [14]. The number of participants per study ranged from 15 to 672 (Mean 151; SD 161). All studies included adults. Minimal to no data was retrieved on paediatric OD.

The two dysphagia-specific HRQoL questionnaires most frequently used were Swallowing Quality-of-Life Questionnaire (SWAL-QOL; 17 studies) [49] and M. D. Anderson Dysphagia Inventory (MDADI; seven studies) [61]. The non-dysphagia-specific HRQoL questionnaire most frequently used was the Short-Form Health Survey (SF-36; four studies) [62].

\section{HRQoL and OD}

This review summarizes data on the relationships between HRQoL (Table 3) and OD and changes in OD and HRQoL following intervention (Table 4). Most of the included articles 
support inverse bidirectional relationships between decreased HRQoL and increased OD severity.

All 16 articles describing the association between HRQoL and OD in Table 3 found statistically significant data supporting inverse bidirectional relationships between decreased HRQoL and increased OD severity [7, 18, 27, 36, 39-42, 46, 48, 49, 52, 53, 56-58]; four of the six articles describing HRQoL using non-dysphagia specific questionnaires confirmed similar relationships $[14,16,50,53]$. The remaining two articles reported inconsistent findings [6] or statistical non-significant findings only [19]. Eight articles included a generic QoL measure [19, 38, 48-51, 54, 57]; all studies confirmed statistically significant inverse relationships between QoL and OD. Finally, three articles reported only mean HRQoL data in patient populations with OD $[26,37,45]$ without reporting on the association with OD severity. Six of the 27 articles in Table 3 objectified the magnitude of the identified relations by determining correlation coefficients $[16,27,40,43,52,57]$. Due to the heterogeneity of the included studies, no weighted means of correlation coefficients were reported.

Eight studies provided pre-post intervention means, SD and $t$ - or $f$-values for changes in both OD severity and HRQoL (Table 4). Five of these studies supported the second hypothesis that a decrease in severity of OD symptoms will lead to a significant increase in HRQoL [34, 43, 55, 60] or QoL [59]. Two studies demonstrated a similar pattern, but with non-significant changes [35, 47]. One study [44] reported improved mean HRQoL after intervention, but did not report on whether the identified changes were statistically significant or not, as data were presented as mean group data without details on those patients that were lost to follow-up (died). Statistical significance of the reported changes could therefore not be determined for review purposes. Only one study reported correlations between the change scores in OD severity and HRQoL [35]. No study conducted a multivariate regression analysis to simultaneously account for the impact of OD severity in the improvement of HR- 
QoL alongside other independent variables that may influence HR-QoL. A meta-analysis could not be conducted due to the heterogeneity of study designs, clinical populations, outcomes and outcome measures used. 
Table 3 The relationships between health-related quality of life and quality of life with oropharyngeal dysphagia

\begin{tabular}{|c|c|c|c|c|}
\hline Literature & $\begin{array}{l}\mathbf{N}, \text { Age in years/ Etiology of } \\
\text { OD }\end{array}$ & $\begin{array}{l}\text { HRQoL to OD, HRQoL, QoL, FHS, OD gold } \\
\text { standard }\end{array}$ & Treatment(s)/ Groups & Key Finding(s)/Conclusion(s) \\
\hline $\begin{array}{l}\text { Airoldi et al., } \\
2011[16]\end{array}$ & $\begin{array}{l}\mathrm{N}=36 \\
(\mathrm{M}=26, \mathrm{~F}=10) \\
\text { Mean age: } 61 \\
\text { Age range: } 31-79 \\
\rightarrow \text { Carcinoma of oral cavity } \\
\text { surgically treated with flap } \\
\text { reconstructive surgery and } \\
\text { adjuvant RT }\end{array}$ & $\begin{array}{l}\text { HRQoL to OD: } \\
\text { NA } \\
\text { HRQoL: } \\
\text { HADS (Hospital Anxiety and Depression Scale) } \\
\text { QLQ-C30 (EORTC Core Questionnaire) } \\
\text { QoL: } \\
\text { NA } \\
\text { FHS: } \\
\text { NA } \\
\text { OD gold standard: } \\
\text { None - confirmed by clinical assessment } \\
\text { OD Measure: } \\
\text { Dische rating scale - participants divided into } \\
\text { severity groups I - V based on Dische scores }\end{array}$ & $\begin{array}{l}\text { Group I: } \\
\text { (N=5; } 14 \%) \\
\text { Dische Score }=0 ; \text { No OD } \\
\text { Group II: } \\
\text { (N=8; } 22 \%) \\
\text { Dische score = } 1 \text {; Some } \\
\text { discomfort swallowing } \\
\text { Group III: } \\
(\mathrm{N}=15 ; 42 \%) \\
\text { Dische score }=2 \text {; Difficulty } \\
\text { with swallowing } \\
\text { Group IV: } \\
\text { (N=4; } 11 \%) \\
\text { Dische Score = } 3 \\
\text { Considerable difficulty } \\
\text { Group V: } \\
\text { (N=4; } 11 \%) \\
\text { Dische score = } 4 ; \text { Severe } \\
\text { difficulty }\end{array}$ & $\begin{array}{l}\rightarrow \text { Group V reported decreased Global Health and QoL (EORTC) scores } \\
\text { compared to Group I and II ( } p<0.001) \text {. This included significantly lower } \\
\text { scores for "Physical Functioning", "Social Functioning", "Fatigue", } \\
\text { "Social Eating", "Social Contacts" and "Sexuality" subscales compared to } \\
\text { groups I and II }(p<0.001) \text {. } \\
\rightarrow \text { Group IV and V reported statistically significant higher levels of } \\
\text { anxiety and depression (HADS) compared to participants in Group I and } \\
\text { to Group II (p }<0.001) \\
\rightarrow \text { Significant correlation identified between dysphagia severity (Dische) } \\
\text { and HADS scores: for depression } r=0.389(p=.019) \text {; for anxiety } r= \\
0.387(p=.02) \text {. }\end{array}$ \\
\hline $\begin{array}{l}\text { Asadollahpour } \\
\text { et al., } 2015[36]\end{array}$ & $\begin{array}{l}\mathrm{N}=174 \\
(\mathrm{M}=\mathrm{NR}, \mathrm{F}=\mathrm{NR}) \\
\text { Mean age: } \mathrm{NR} \\
\text { Age range: } \mathrm{NR} \\
\text { Group I: } \\
\mathrm{N}=85 \\
\text { (M=NR, F=NR) } \\
\text { Mean age: } 62 \\
\text { Age range: } 37-80 \\
\rightarrow \text { Participants with OD due to } \\
\text { HNC, stroke, ALS, PD and } \\
\text { esophageal achalasia } \\
\text { Group II: } \\
\mathrm{N}=89 \\
\text { (M=47, F=42) } \\
\text { Mean age: } 64 \\
\text { Age range: } 45-83 \\
\rightarrow \text { No OD (age- and gender- } \\
\text { matched healthy controls) }\end{array}$ & $\begin{array}{l}\text { HRQoL to OD: } \\
\text { P-DHI (Persian Dysphagia Handicap Index) } \\
\text { HRQoL: } \\
\text { NA } \\
\text { QoL: } \\
\text { NA } \\
\text { FHS: } \\
\text { NA } \\
\text { OD gold standard: } \\
\text { NR. - diagnosed by a laryngologist } \\
\text { OD measure: } \\
\text { Diagnosed by a laryngologist }\end{array}$ & $\begin{array}{l}\rightarrow \text { All participants completed } \\
\text { the questionnaire } 2 \text { w. apart. }\end{array}$ & $\begin{array}{l}\rightarrow \text { Group I presented with statistically significant lower QoL (P-DHI) } \\
\text { compared with Group II }(p<0.001) \text {. }\end{array}$ \\
\hline $\begin{array}{l}\text { Bibi el al., } 2015 \\
\text { [37] }\end{array}$ & $\begin{array}{l}\mathrm{N}=40 \\
(\mathrm{M}=24, \mathrm{~F}=16) \\
\text { Mean age: } \mathrm{NR} \\
\text { Age range: } \mathrm{NR}\end{array}$ & $\begin{array}{l}\text { HRQoL to OD: } \\
\text { SWAL-QOL (Swallowing Quality of Life } \\
\text { Questionnaire - Urdu-translated version) }\end{array}$ & $\begin{array}{l}\rightarrow \text { All participants completed } \\
\text { questionnaire. }\end{array}$ & $\begin{array}{l}\rightarrow \text { Mean HRQoL scores for study participants are presented. No other } \\
\text { relevant data available. }\end{array}$ \\
\hline
\end{tabular}




\begin{tabular}{|c|c|c|c|c|}
\hline Literature & $\begin{array}{l}\mathrm{N}, \text { Age in years/ Etiology of } \\
\text { OD }\end{array}$ & $\begin{array}{l}\text { HRQoL to OD, HRQoL, QoL, FHS, OD gold } \\
\text { standard }\end{array}$ & Treatment(s)/ Groups & Key Finding(s)/Conclusion(s) \\
\hline & $\begin{array}{l}\rightarrow \text { Participants with OD due to } \\
\text { stroke }(47.5 \%), \text { PD }(22.5 \%), \\
\text { TBI }(20 \%) \text {, Injury }(7.5 \%), \text { HNC } \\
(2.5 \%) \text { and other causes }\end{array}$ & $\begin{array}{l}\text { HRQoL: } \\
\text { NA } \\
\text { QoL: } \\
\text { None } \\
\text { FHS: } \\
\text { None } \\
\text { OD gold standard: } \\
\text { NR } \\
\text { OD measure: } \\
\text { NR }\end{array}$ & & \\
\hline $\begin{array}{l}\text { Bogaardt et al., } \\
2009[26]\end{array}$ & $\begin{array}{l}\mathrm{N}=152 \\
(\mathrm{M}=99, \mathrm{~F}=53) \\
\text { Mean age: } 65 \\
\text { Age range: } 20-91 \\
\rightarrow \text { Participants with OD due to } \\
\text { stroke }(28 \%), \mathrm{PD}(24 \%), \\
\text { NMD }(9 \%) \text {, other neurologic } \\
\text { diseases }(16 \%), \text { HNC }(11 \%), \\
\text { Idiopathic cricopharyngeal } \\
\text { problems (5\%), unknown ( } 7 \%)\end{array}$ & $\begin{array}{l}\text { HRQoL to OD: } \\
\text { SWAL-QOL-NL (Swallowing Quality of Life } \\
\text { Questionnaire - Dutch version) } \\
\text { HRQoL: } \\
\text { None } \\
\text { QoL: } \\
\text { EQ-5D (EuroQol-5D Questionnaire) } \\
\text { FHS: } \\
\text { None } \\
\text { OD gold standard: } \\
\text { None - confirmed by clinical assessment } \\
\text { OD measure: } \\
\text { Clinical assessment }\end{array}$ & $\begin{array}{l}\text { All participants completed } \\
\text { both questionnaires. }\end{array}$ & $\begin{array}{l}\rightarrow \text { Mean HRQoL scores (SWAL-QOL) for study participants are } \\
\text { presented. No other relevant data available. }\end{array}$ \\
\hline $\begin{array}{l}\text { Campbell et al., } \\
2004[38]\end{array}$ & $\begin{array}{l}\mathrm{N}=62 \\
(\mathrm{M}=45, \mathrm{~F}=17) \\
\text { Mean age: } \mathrm{NR} \\
\text { Age range: } \mathrm{NR} \\
\rightarrow 5 \text {-yr survivors of HNC (non- } \\
\text { laryngectomy) with aspiration } \\
\text { or no aspiration }\end{array}$ & $\begin{array}{l}\text { HRQoL to OD: } \\
\text { NA } \\
\text { HRQoL } \\
\text { NA } \\
\text { QoL: } \\
\text { UWQOL (University of Washington Quality of Life } \\
\text { Scale) } \\
\text { FHS: } \\
\text { PSS-HN (Performance Status Scale for Head and } \\
\text { Neck Cancer Participants) }\end{array}$ & $\begin{array}{l}\text { Group I: } \\
(\mathrm{N}=27 ; 44 \%) \\
\text { Aspirators } \\
\text { Group II: } \\
(\mathrm{N}=35 ; 56 \%) \\
\text { Non-aspirators }\end{array}$ & $\begin{array}{l}\rightarrow \text { Group I scored statistically significant lower on QoL (UWQOL) for } \\
\text { "Chewing" }(p=0.002) \text { and "Swallowing" }(p=0.04) \text {; but no significant } \\
\text { difference between groups for other } 7 \text { subscales } \\
\rightarrow \text { Group I scored significantly lower FHS (PSS-HN) for "Normalcy of } \\
\text { diet" }(p=0.01) \text {; but no significant difference between groups for other } 2 \\
\text { subscales. } \\
\rightarrow \text { No significant between group differences for FACT-G Wellbeing } \\
\text { Scales } \\
\rightarrow \text { Group I scored significantly lower on FACT-HN for "Additional } \\
\text { Concerns" }(p=0.01)\end{array}$ \\
\hline
\end{tabular}




\begin{tabular}{|c|c|c|c|c|}
\hline Literature & $\begin{array}{l}\mathrm{N}, \text { Age in years/ Etiology of } \\
\text { OD }\end{array}$ & $\begin{array}{l}\text { HRQoL to OD, HRQoL, QoL, FHS, OD gold } \\
\text { standard }\end{array}$ & Treatment(s)/ Groups & Key Finding(s)/Conclusion(s) \\
\hline & & $\begin{array}{l}\text { FACT-G (Functional Assessment of Cancer } \\
\text { Treatment - General) } \\
\text { FACT-H\&N (Functional Assessment of Cancer } \\
\text { Therapy - Head and Neck Scale) } \\
\text { OD gold standard: } \\
\text { VFS } \\
\text { OD measure: } \\
\text { VFS used to classify as "aspirators" or "non- } \\
\text { aspirators" }\end{array}$ & & \\
\hline $\begin{array}{l}\text { Carlsson et al., } \\
2012[39]\end{array}$ & $\begin{array}{l}\mathrm{N}=230 \\
\text { (M=132, F=98) } \\
\text { Mean age: NR } \\
\text { Age range: } 37-92 \\
\text { Group I: } \\
\text { Participants with OD including: } \\
\text { Neurologic disease with OD: } \\
\mathrm{N}=30 \\
\text { (M=14, F=16) } \\
\text { Mean age: } 68 \\
\text { Age range: } 42-88 \\
\text { HNC with curative intent and } \\
\text { post-treatment subjective OD } \\
\mathrm{N}=85 \\
\text { (M=52, F=33) } \\
\text { Mean age: } 63 \\
\text { Age range: } 37-92 \\
\text { Group II: } \\
\mathrm{N}=115 \\
\text { (M=66, F=49) } \\
\text { Mean age: } 63 \\
\text { Age range: } 31-89 \\
\rightarrow \text { No OD (healthy controls) } \\
\end{array}$ & $\begin{array}{l}\text { HRQoL to OD: } \\
\text { MDADI (M.D. Anderson Dysphagia Inventory - } \\
\text { Swedish Version) } \\
\text { HRQoL: } \\
\text { NA } \\
\text { QoL: } \\
\text { NA } \\
\text { FHS: } \\
\text { NA } \\
\text { OD gold standard: } \\
\text { None - confirmed by clinical assessment } \\
\text { OD measure: } \\
\text { Self-report }\end{array}$ & $\begin{array}{l}\text { Group I: } \\
\text { (N=115; 50\%) } \\
\text { Participants with OD } \\
\text { Includes: } \\
\text { Neurological participants } \\
\text { (N=30; } 13 \%) \\
\text { HNC participants (N=85; } \\
37 \%) \\
\text { Group II: } \\
\text { (N=115; } 50 \%) \\
\text { No OD }\end{array}$ & $\begin{array}{l}\rightarrow \text { Group I scored statistically significant lower on HRQoL (MDADI) for } \\
\text { all domains compared with Group II ( } p<0.001 \text { ) } \\
\rightarrow \text { Within Group I, tube-fed participants with OD scored significantly } \\
\text { lower on HRQoL (MDADI) for all domains except "physical" domain in } \\
\text { comparison to participants with OD on a regular diet ( } p \leq 0.05 \text { ); and } \\
\text { significantly lower HRQoL (MDADI) for all domains except "emotional" } \\
\text { in comparison to participants with OD on a soft-food diet }(p \leq 0.05 \text {. }\end{array}$ \\
\hline $\begin{array}{l}\text { da Costa } \\
\text { Franceschini et } \\
\text { al., 2015 [40] }\end{array}$ & $\begin{array}{l}\mathrm{N}=17 \\
(\mathrm{M}=11, \mathrm{~F}=6) \\
\text { Mean age: } 56 \\
\text { Age range: } 31-69 \\
\rightarrow \text { Participants with OD due to } \\
\text { ALS with spinal onset } \\
\text { Group I } \\
\mathrm{N}=5 \\
\text { (M=NR, F=NR) } \\
\text { Mean age: } \mathrm{NR}\end{array}$ & $\begin{array}{l}\text { HRQoL to OD: } \\
\text { SWAL-QOL (Swallowing Quality of Life } \\
\text { Questionnaire - Urdu-translated version) } \\
\text { HRQoL: } \\
\text { NA } \\
\text { QoL: } \\
\text { NA } \\
\text { FHS: }\end{array}$ & $\begin{array}{l}\rightarrow \text { All participants completed } \\
\text { both questionnaires. } \\
\text { Group I: Do } \\
\text { Participants without OD } \\
\text { Group II: D1 } \\
\text { Participants with mild OD } \\
\text { Group III: D2 } \\
\text { Participants with moderate } \\
\text { OD } \\
\text { Group IV: D3 }\end{array}$ & $\begin{array}{l}\rightarrow \text { Significant correlations were identified between HRQoL (SWALQOL } \\
\text { domain scores) and dysphagia severity (based on FEES and FOIS); } \\
\text { indicating association between severe dysphagia and lower QoL. } \\
\rightarrow \text { Correlations between dysphagia severity versus SWALQOL domains } \\
\text { "Symptom Frequency", "Communication" and "Fear" were, respectively, } \\
r=-0.715(p<0.001), r=-0.673(p=0.003) \text { and } r=-0.657(p= \\
0.004) \text {; correlations between FOIS versus SWALQOL domains } \\
\text { "Symptom Frequency", "Communication", "Fear" and "Burden" were, } \\
\text { respectively, } r=0.583 \text { ( }(p=0.014), r=0.644(p=0.005), r=0.578 \text { ( } p= \\
0.015) \text { and } r=-0.570(p=0.017) \text {. }\end{array}$ \\
\hline
\end{tabular}




\begin{tabular}{|c|c|c|c|c|}
\hline Literature & $\begin{array}{l}\mathrm{N}, \text { Age in years/ Etiology of } \\
\text { OD }\end{array}$ & $\begin{array}{l}\text { HRQoL to OD, HRQoL, QoL, FHS, OD gold } \\
\text { standard }\end{array}$ & Treatment(s)/ Groups & Key Finding(s)/Conclusion(s) \\
\hline & $\begin{array}{l}\text { Age range: } \mathrm{NR} \\
\rightarrow \text { Participants without } \mathrm{OD} \\
\text { Group II } \\
\mathrm{N}=7 \\
(\mathrm{M}=\mathrm{NR}, \mathrm{F}=\mathrm{NR}) \\
\text { Mean age: NR } \\
\text { Age range: } \mathrm{NR} \\
\rightarrow \text { Participants with mild OD } \\
\text { Group III } \\
\mathrm{N}=5 \\
(\mathrm{M}=\mathrm{NR}, \mathrm{F}=\mathrm{NR}) \\
\text { Mean age: NR } \\
\text { Age range: } \mathrm{NR} \\
\rightarrow \text { Participants with moderate } \\
\text { OD } \\
\text { Group IV } \\
\mathrm{N}=0 \\
(\mathrm{M}=0, \mathrm{~F}=0) \\
\text { Mean age: } 0 \\
\text { Age range: } 0 \\
\rightarrow \text { Participants with severe } \mathrm{OD}\end{array}$ & $\begin{array}{l}\text { FOIS (Functional Oral Intake Scale): Participants } \\
\text { graded across } 7 \text { levels: - Level 1: Nil by mouth - } \\
\text { Level 7: total oral diet with no restrictions } \\
\text { OD gold standard: } \\
\text { FEES } \\
\text { OD measure: } \\
\text { Based on FEES, participants were identified by group } \\
\text { I, II, III or IV. }\end{array}$ & Participants with severe OD & $\begin{array}{l}\rightarrow \text { Group III reported significantly lower QoL (SWALQOL) than Group I } \\
\text { for "Symptom Frequency" }(p=0.008) \text {, "Communication" }(p=0.016) \text { and } \\
\text { "Fear" }(p=0.025) \text {. Group III reported significantly lower QoL (SWAL- } \\
\text { QoL) than Group II for "Symptom Frequency" }(p=0.018) \text {, "Food } \\
\text { Selection" }(p=0.025) \text {, "Communication" }(p=0.038) \text { and "Fear" }(p= \\
\text { 0.03). There were no statistical group differences identified for other } \\
\text { SWALQOL domains and no reported differences between Groups I \& II. }\end{array}$ \\
\hline $\begin{array}{l}\text { da Silva } \\
\text { Brandão et al., } \\
2010[6]\end{array}$ & $\begin{array}{l}\mathrm{N}=60 \\
(\mathrm{M}=35, \mathrm{~F}=25) \\
\rightarrow \text { Stroke } \\
\text { Group I: } \\
\mathrm{N}=30 \\
(\mathrm{M}=\mathrm{NR}, \mathrm{F}=\mathrm{NR}) \\
\text { Age: } 60 \text { and over } \\
\rightarrow \text { OD } \\
\text { Group II: } \\
\mathrm{N}=30 \\
(\mathrm{M}=\mathrm{NR}, \mathrm{F}=\mathrm{NR}) \\
\text { Mean age: } \mathrm{NR} \\
\text { Age range: NR } \\
\rightarrow \text { No OD }\end{array}$ & $\begin{array}{l}\text { HRQoL to OD: } \\
\text { NA } \\
\text { HRQoL: } \\
\text { SF-36 (Short-Form Health Survey - Brazilian } \\
\text { version) } \\
\text { QoL: } \\
\text { NA } \\
\text { FHS: } \\
\text { ADL (Basic Activities of Daily Living scale - also } \\
\text { called Katz Functional Assessment) } \\
\text { Lawton - IADL scale (Instrumental Activities of } \\
\text { Daily Living) } \\
\text { Barthel Index (Activities of Daily Living) } \\
\text { OD gold standard: } \\
\text { None - confirmed by clinical assessment } \\
\text { OD measure: } \\
\text { Clinical assessment }\end{array}$ & $\begin{array}{l}\text { Group I: } \\
(\mathrm{N}=30 ; 50 \%) \\
\text { OD } \\
\text { Group II: } \\
(\mathrm{N}=30 ; 50 \%) \\
\text { No OD } \\
\rightarrow \text { QoL questionnaires } \\
\text { completed 3-12 m. post- } \\
\text { stroke. }\end{array}$ & $\begin{array}{l}\rightarrow \text { No significant between group differences for FHS (ADL, Lawton } \\
\text { IADL and Barthel Index) } \\
\rightarrow \text { HRQoL (SF-36) scores indicated that Group I exhibited significantly } \\
\text { less Pain }(p=0.040) \text { but significantly worse General Health }(p=0.017) \\
\text { than Group II. There were no between groups differences on any other } \\
\text { SF-36 domains. }\end{array}$ \\
\hline $\begin{array}{l}\text { dos Santos } \\
\text { Queija et al., } \\
2009 \text { [41] }\end{array}$ & $\begin{array}{l}\mathrm{N}=28 \\
(\mathrm{M}=26, \mathrm{~F}=2) \\
\text { Mean age: } 59\end{array}$ & $\begin{array}{l}\text { HRQoL to OD: } \\
\text { SWAL-QOL (Swallowing Quality of Life } \\
\text { Questionnaire) }\end{array}$ & $\begin{array}{l}\text { Group I: } \\
(\mathrm{N}=10 ; 36 \%) \\
\text { No OD }\end{array}$ & $\begin{array}{l}\rightarrow \text { Stratified analysis of complaints and SWAL-QOL indicated a greater } \\
\text { QoL impact associated with swallowing in those patients who reported } \\
\text { swallowing difficulties (Group II and III): significant differences in }\end{array}$ \\
\hline
\end{tabular}




\begin{tabular}{|c|c|c|c|c|}
\hline Literature & $\begin{array}{l}\mathrm{N}, \text { Age in years/ Etiology of } \\
\text { OD }\end{array}$ & $\begin{array}{l}\text { HRQoL to OD, HRQoL, QoL, FHS, OD gold } \\
\text { standard }\end{array}$ & Treatment(s)/ Groups & Key Finding(s)/Conclusion(s) \\
\hline & $\begin{array}{l}\text { Age range: } 42-82 \\
\rightarrow \text { Total laryngectomy }(\mathrm{N}=15) \\
\text { and total pharyngo- } \\
\text { laryngectomy }(\mathrm{N}=13)\end{array}$ & $\begin{array}{l}\text { HRQoL: } \\
\text { NA } \\
\text { QoL: } \\
\text { NA } \\
\text { FHS: } \\
\text { NA } \\
\text { OD gold standard: } \\
\text { VFS } \\
\text { OD measure: } \\
\text { Based on VFS, participants were classified by } \\
\text { severity }\end{array}$ & $\begin{array}{l}\text { Group II: } \\
(\mathrm{N}=12 ; 43 \%) \\
\text { Mild OD } \\
\text { Group III: } \\
(\mathrm{N}=6 ; 21 \%) \\
\text { Moderate-severe OD }\end{array}$ & $\begin{array}{l}\text { subscales "Burden" }(p=0.036 \text { and "Mental Health" }(p=0.031) \text {. Further } \\
\text { analysis to identify differences among the groups, showed a significant } \\
\text { difference between Group I versus Group II-III }(p=0.12) \text {. }\end{array}$ \\
\hline $\begin{array}{l}\text { Eslick \& Talley, } \\
2008[14]\end{array}$ & $\begin{array}{l}\mathrm{N}=672 \\
(\mathrm{M}=322, \mathrm{~F}=350) \\
\text { Mean age: NR } \\
\text { Age range: NR } \\
\text { Group I: } \\
\mathrm{N}=110 \\
\text { (M=NR, F=NR) } \\
\text { Mean age: NR } \\
\text { Age range: NR } \\
\rightarrow \text { OD } \\
\text { Group II: } \\
\mathrm{N}=562 \\
\text { (M=NR, F=NR) } \\
\text { Mean age: NR } \\
\text { Age range: NR } \\
\rightarrow \text { No OD (controls) }\end{array}$ & $\begin{array}{l}\text { HRQoL to OD: } \\
\text { NA } \\
\text { HRQoL: } \\
\text { SF-36 (Short-Form Health Survey) } \\
\text { QoL: } \\
\text { NA } \\
\text { FHS: } \\
\text { NA } \\
\text { OD gold standard: } \\
\text { None - confirmed by self-report (Chest Pain } \\
\text { Questionnaire) } \\
\text { OD Measure: } \\
\text { Presence and severity of dysphagia determined by } \\
\text { self-report (Chest Pain Questionnaire) }\end{array}$ & $\begin{array}{l}\text { Group I: } \\
(\mathrm{N}=110 ; 16 \%) \\
\text { mild OD }=65 \% ; \\
\text { moderate }=30 \% \\
\text { Severe = 5\% } \\
\text { Group II: } \\
\text { (N=562; 73\%) } \\
\text { No OD }\end{array}$ & $\begin{array}{l}\rightarrow \text { Group I compared to Group II (no OD) scored statistically significant } \\
\text { lower QoL (SF-36: 7/8 subscales } p<0.04) \text {. } \\
\rightarrow \text { Subgroups I: patients with moderate OD compared to mild OD scored } \\
\text { significantly lower QoL on "Physical Functioning" ( } p=0.03) \text {; patients } \\
\text { with very severe OD compared to severe OD scored significantly lower } \\
\text { QoL on "Vitality" ( } p=0.03 \text { ), "Social Functioning" ( } p=0.03 \text { ) and "Role } \\
\text { Emotional" ( }(p<0.001) \text {. No statistical differences for other subgroup } \\
\text { comparisons. } \\
\rightarrow \text { Intermittent dysphagia was independently associated with anxiety (OR } \\
=1.09,95 \% \text { CI: } \\
1.01-1.19) \text { The presence of progressive dysphagia was independently } \\
\text { associated with depression (OR }=1.34,95 \% \text { CI: } 1.07-1.67) \text {. } \\
\rightarrow \text { Progressive dysphagia was independently associated with reduced } \\
\text { "general health" (OR }=0.95,95 \% \text { CI: } 0.90-0.99) \text {, while intermittent } \\
\text { dysphagia was associated with a reduction in the "role physical" subscale } \\
\text { (OR = } 0.98,95 \% \text { CI: } 0.97-0.99) \text {. }\end{array}$ \\
\hline $\begin{array}{l}\text { Finizia et al., } \\
2012[39]\end{array}$ & $\begin{array}{l}\mathrm{N}=230 \\
(\mathrm{M}=\mathrm{NR}, \mathrm{F}=\mathrm{NR}) \\
\text { Mean age: } \mathrm{NR} \\
\text { Age range: } \mathrm{NR} \\
\text { Group I: } \\
\mathrm{N}=115 \\
(\mathrm{M}=66, \mathrm{~F}=49) \\
\text { Mean age: } 64 \\
\text { Age range: } 37-92\end{array}$ & $\begin{array}{l}\text { HRQoL to OD: } \\
\text { SWAL-QOL (Swallowing Quality of Life } \\
\text { Questionnaire - Swedish version), } \\
\text { HRQoL: } \\
\text { NA } \\
\text { QoL: } \\
\text { NA } \\
\text { FHS: }\end{array}$ & $\begin{array}{l}\text { Group Ia: } \\
\text { (N=30; } 26 \%) \\
\text { Neurological disease with } \\
\text { OD. } \\
\rightarrow \text { Completion of } \\
\text { questionnaire within } 0.2-1.5 \\
\text { yr post-diagnosis. } \\
\text { Group Ib: } \\
\text { (N=85; } 74 \% \text { ) } \\
\text { HNC with OD. }\end{array}$ & $\begin{array}{l}\rightarrow \text { Group I reported significantly lower HRQoL (SWAL-QOL) }(p< \\
0.000) \text { compared with Group II. } \\
\rightarrow \text { Significant between group differences were found for total SWAL- } \\
\text { QOL score between participants on a regular vs soft diet }(p<0.05) \text {; } \\
\text { regular vs pureed diet }(p<0.05) \text {; regular diet vs tube-fed }(p<0.001) \text {; soft } \\
\text { diet vs tube-fed }(p<0.01) \text {. }\end{array}$ \\
\hline
\end{tabular}




\begin{tabular}{|c|c|c|c|c|}
\hline Literature & $\begin{array}{l}\mathbf{N}, \text { Age in years/ Etiology of } \\
\text { OD }\end{array}$ & $\begin{array}{l}\text { HRQoL to OD, HRQoL, QoL, FHS, OD gold } \\
\text { standard }\end{array}$ & Treatment(s)/Groups & Key Finding(s)/Conclusion(s) \\
\hline & $\begin{array}{l}\rightarrow \text { HNC with OD }(\mathrm{N}=85), \\
\text { neurological disease with } \mathrm{OD} \\
(\mathrm{N}=30) \\
\text { Group II: } \\
\mathrm{N}=115 \\
(\mathrm{M}=66, \mathrm{~F}=49) \\
\text { Mean age: } 63 \\
\text { Age range: } 31-89 \\
\rightarrow \text { No OD (age- and gender- } \\
\text { matched healthy controls) }\end{array}$ & $\begin{array}{l}\text { FOIS (Functional Oral Intake Scale) } \\
\text { OD gold standard: } \\
\text { FEES \& VFS }(\mathrm{N}=12 \text { HNC) and confirmed by self- } \\
\text { assessment }(\mathrm{N}=218) \\
\text { OD measure: } \\
\text { Presence and severity of dysphagia determined by } \\
\text { self-report }\end{array}$ & $\begin{array}{l}\rightarrow \text { Completion of } \\
\text { questionnaire within } 0.3-2.9 \\
\text { yr post-treatment. } \\
\text { Group II: } \\
(\mathrm{N}=115) \\
\text { No OD }\end{array}$ & \\
\hline $\begin{array}{l}\text { Florie et al., } \\
2015[42]\end{array}$ & $\begin{array}{l}\mathrm{N}=63 \\
(\mathrm{M}=51, \mathrm{~F}=12) \\
\text { Mean age: } 66 \\
\text { Age range: } \mathrm{NR} \\
\rightarrow \text { Participants with OD due to } \\
\text { HNC }\end{array}$ & $\begin{array}{l}\text { HRQoL to OD: } \\
\text { MDADI (MD Anderson Dysphagia Inventory) } \\
\text { HRQoL: } \\
\text { NA } \\
\text { QoL: } \\
\text { NA } \\
\text { FHS: } \\
\text { NA } \\
\text { OD gold standard: } \\
\text { FEES } \\
\text { OD measure: } \\
\text { FEES (Dysphagia severity categorised by clinician) }\end{array}$ & $\begin{array}{l}\rightarrow \text { All participants completed } \\
\text { questionnaire and underwent } \\
\text { FEES. }\end{array}$ & $\begin{array}{l}\rightarrow \text { Only } 3 / 60 \text { analyses comparing mean differences on MDADI subscale } \\
\text { ratings between ordinal categories of FEES variables ( } 3 \text { consistencies) } \\
\text { resulted in statistically significant data: } \\
\text { MDADI -Physical scale with piecemeal deglutition ( } p=0.043 \text { ), and } \\
\text { MDADI-General and MDADI-Functional subscales with post-swallow } \\
\text { vallecular pooling (resp., } p=0.020 \text { and } p=0.018 \text { ) for thick liquid. These } \\
\text { results indicated that worse swallowing functioning was associated with } \\
\text { lower HRQoL. } \\
\rightarrow \text { All other comparisons showed no statistically significant results. }\end{array}$ \\
\hline $\begin{array}{l}\text { García-Peris et } \\
\text { al., } 2007[18]\end{array}$ & $\begin{array}{l}\mathrm{N}=87 \\
(\mathrm{M}=61, \mathrm{~F}=26) \\
\text { Mean age: } 58 \\
\text { Age range: } 16-82 \\
\rightarrow \text { HNC treated with surgery } \\
\text { and RT or CT }(28.5+/-17.8 \mathrm{~m} \\
\text { post-surgery) }\end{array}$ & $\begin{array}{l}\text { HRQoL to OD: } \\
\text { European Dysphagia Group Questionnaire (Part 3: } \\
\text { "Personal feelings and importance") } \\
\text { HRQoL: } \\
\text { NA } \\
\text { QoL: } \\
\text { NA } \\
\text { FHS: } \\
\text { NA } \\
\text { OD gold standard: } \\
\text { None - determined by self-report (Questionnaire) } \\
\text { OD measure: } \\
\text { Self-report }\end{array}$ & $\begin{array}{l}\text { Group I: } \\
\text { (N=44; } 51 \%) \\
\text { OD } \\
\text { Group II: } \\
\text { (N=43; 49\%) } \\
\text { No OD }\end{array}$ & $\begin{array}{l}\rightarrow \text { Group I reported significantly lower HRQoL (European Dysphagia } \\
\text { Group Questionnaire) compared with Group II ( } p<0.05 \text { ). }\end{array}$ \\
\hline
\end{tabular}




\begin{tabular}{|c|c|c|c|c|}
\hline Literature & $\begin{array}{l}\mathrm{N}, \text { Age in years/ Etiology of } \\
\text { OD }\end{array}$ & $\begin{array}{l}\text { HRQoL to OD, HRQoL, QoL, FHS, OD gold } \\
\text { standard }\end{array}$ & Treatment(s)/ Groups & Key Finding(s)/Conclusion(s) \\
\hline $\begin{array}{l}\text { Lam \& Lai, } 2011 \\
{[45]}\end{array}$ & $\begin{array}{l}\mathrm{N}=100 \\
(\mathrm{M}=63, \mathrm{~F}=37) \\
\text { Mean age: } 60 \\
\text { Age range: } 47-74 \\
\rightarrow \text { Mechanical or neurological } \\
\text { OD. }\end{array}$ & $\begin{array}{l}\text { HRQoL to OD: } \\
\text { CSWAL-QOL (Swallowing Quality of Life } \\
\text { Questionnaire - Chinese Version) } \\
\text { HRQoL: } \\
\text { WHOQOL-BREF [HK] (World Health } \\
\text { Organization Quality of Life Measure-abbreviated } \\
\text { version, Hong Kong) } \\
\text { QoL: } \\
\text { NA } \\
\text { FHS: } \\
\text { NA } \\
\text { OD gold standard: } \\
\text { None - confirmed by clinical assessment (bedside } \\
\text { swallowing test) }\end{array}$ & $\begin{array}{l}\text { All participants completed } \\
\text { both questionnaires. }\end{array}$ & $\begin{array}{l}\rightarrow \text { Mean HRQoL scores (SWAL-QOL) for study participants are } \\
\text { presented. No other relevant data available. }\end{array}$ \\
\hline $\begin{array}{l}\text { Leow et al., } 2010 \\
{[46]}\end{array}$ & $\begin{array}{l}\mathrm{N}=64 \\
(\mathrm{M}=48, \mathrm{~F}=16) \\
\text { Mean age: NR } \\
\text { Age range: } \mathrm{NR} \\
\\
\text { Group I: } \\
\mathrm{N}=16 \\
\text { (M=8, F=8) } \\
\text { Mean age: } 25 \\
\text { Age range: } 21-32 \\
\rightarrow \text { No OD (healthy adult } \\
\text { controls) } \\
\text { Group II: } \\
\mathrm{N}=16 \\
\text { (M=8, F=8) } \\
\text { Mean age: } 73 \\
\text { Age range: } 62-85 \\
\rightarrow \text { No OD (healthy elder } \\
\text { controls) } \\
\text { Group III: } \\
\text { N=32 } \\
\text { (M=32) } \\
\text { Mean age: } 69 \\
\text { Age range: } 46-83 \\
\rightarrow \text { IPD } \\
\end{array}$ & $\begin{array}{l}\text { HRQoL to OD: } \\
\text { SWAL-QOL (Swallowing Quality of Life } \\
\text { Questionnaire) } \\
\text { HRQoL: } \\
\text { NA } \\
\text { QoL: } \\
\text { NA } \\
\text { FHS: } \\
\text { NA } \\
\text { OD gold standard: } \\
\text { None }\end{array}$ & $\begin{array}{l}\text { Group I: } \\
\text { (N=16; 25\%) } \\
\text { Healthy adult controls } \\
\text { Group II: } \\
\text { (N=16; 25\%) } \\
\text { Healthy elder controls } \\
\text { Group IIIa: } \\
\text { (N=16; 25\%) } \\
\text { HY } \leq \text { stage 2 } \\
\text { Group IIIb: } \\
\text { (N=16; 25\%) } \\
\text { HY } \geq \text { stage 2 }\end{array}$ & $\begin{array}{l}\rightarrow \text { Group III reported significantly lower HRQoL (SWAL-QOL) } \\
\text { compared with Group II on all subscales except "Sleep" }(p<0.05) \text {. } \\
\rightarrow \text { Significant group differences between Group IIIa and Group IIIb on } \\
\text { SWAL-QoL subscales "Food Selection" ( }<<0.014) \text {, "Eating Duration" } \\
(p<0.010) \text {, "Eating Desire" }(p<0.037) \text { and overall SWAL-QOL score ( } p \\
<0.018) \text {; group IIIb reported lower HRQoL compared to group IIIa. }\end{array}$ \\
\hline $\begin{array}{l}\text { Lovell et al., } \\
2005[48]\end{array}$ & $\begin{array}{l}\mathrm{N}=51 \\
(\mathrm{M}=40, \mathrm{~F}=11)^{\mathrm{b}} \\
\text { Mean age: } 46\end{array}$ & $\begin{array}{l}\text { HRQoL to OD: } \\
\text { SWAL-QOL (Swallowing Quality of Life } \\
\text { Questionnaire) }\end{array}$ & $\begin{array}{l}\text { Group I: } \\
(\mathrm{N}=43 ; 84 \%) \\
\mathrm{OD}\end{array}$ & $\begin{array}{l}\rightarrow \text { Statistically significant association between reported swallowing } \\
\text { difficulties and UW-QOL scores for "swallowing" }(p=0.006) \text { and }\end{array}$ \\
\hline
\end{tabular}




\begin{tabular}{|c|c|c|c|c|}
\hline Literature & $\begin{array}{l}\mathrm{N}, \text { Age in years/ Etiology of } \\
\text { OD }\end{array}$ & $\begin{array}{l}\text { HRQoL to OD, HRQoL, QoL, FHS, OD gold } \\
\text { standard }\end{array}$ & Treatment(s)/ Groups & Key Finding(s)/Conclusion(s) \\
\hline & $\begin{array}{l}\text { Age range: } 16-72 \\
\rightarrow \text { NPC disease-free } \\
\text { participants. }\end{array}$ & $\begin{array}{l}\text { HRQoL: } \\
\text { NA } \\
\text { QoL: } \\
\text { UW-QOL (University of Washington Quality of Life } \\
\text { Questionnaire) } \\
\text { FHS: } \\
\text { NA - determined by patient report } \\
\text { OD gold standard: } \\
\text { None - confirmed by self-assessment }\end{array}$ & $\begin{array}{l}\text { Group II: } \\
\text { (N=8; 16\%) } \\
\text { No OD } \\
\rightarrow \text { Participants selected "no } \\
\text { swallowing difficulty" or } \\
\text { "swallowing problem" from } \\
\text { questionnaire instruction } \\
\text { sheets. } \\
\rightarrow \text { All participants who } \\
\text { indicated a swallowing } \\
\text { problem completed the } \\
\text { SWAL-QOL questionnaire. }\end{array}$ & $\begin{array}{l}\text { "chewing" ( } p=0.118) \text {. No significant associations found for other } \\
\text { swallow-related UW-QOL scores. } \\
\rightarrow \text { Group I reported a lower UW-QOL composite score }(p=0.002) \text { and a } \\
\text { lower SWAL-QOL score compared with Group II }(p=0.004) \text {. } \\
\rightarrow \text { Self-reported swallowing difficulty predicted a lower SWAL-QOL } \\
\text { score }(p=0.004) .\end{array}$ \\
\hline $\begin{array}{l}\text { Maclean et al., } \\
2009[19]\end{array}$ & $\begin{array}{l}\mathrm{N}=110 \\
(\mathrm{M}=98, \mathrm{~F}=12) \\
\text { Mean age: } \mathrm{NR} \\
\text { Age range: } \mathrm{NR} \\
\rightarrow \text { HNC with total } \\
\text { laryngectomy } \\
\text { Group I: } \\
\mathrm{N}=31 \\
\text { (M=NR, F=NR) } \\
\text { Mean age: } \mathrm{NR} \\
\text { Age range: } \mathrm{NR} \\
\rightarrow \text { No OD (healthy controls) } \\
\text { Group II: } \\
\mathrm{N}=79 \\
\text { (M=NR, F=NR) } \\
\text { Mean age: NR } \\
\text { Age range: NR } \\
\rightarrow \text { OD }\end{array}$ & $\begin{array}{l}\text { HRQoL to OD: } \\
\text { NA } \\
\text { HRQoL: } \\
\text { WHOQOL-BREF (World Health Organisation } \\
\text { Quality of Life-BREF) } \\
\text { QoL } \\
\text { UW-QOL (University of Washington Quality of Life } \\
\text { Questionnaire) } \\
\text { DASS (Depression Anxiety and Stress Scale) } \\
\text { FHS: } \\
\text { NA } \\
\text { OD gold standard: } \\
\text { None - confirmed by self-assessment }\end{array}$ & $\begin{array}{l}\text { Group I: } \\
\text { (N=79;72\%) } \\
\text { No OD } \\
\text { Group II: } \\
(\mathrm{N}=31 ; 28 \%) \\
\text { OD }\end{array}$ & $\begin{array}{l}\rightarrow \text { No significant between group differences }(p>0.05) \text { were found for } \\
\text { WHOQOL-BREF scores. All participants (with and without OD) } \\
\text { recorded significantly lower WHOQOL-BREF scores compared with } \\
\text { Australian population norms }(p<0.001 \text { ). } \\
\rightarrow \text { Group II reported significantly lower QoL (UW-QOL) and Depression } \\
\text { and Anxiety (DASS) compared with Group I ( } p<0.05 \text { ). }\end{array}$ \\
\hline $\begin{array}{l}\text { McHorney et al., } \\
2002[49]\end{array}$ & $\begin{array}{l}\mathrm{N}=426 \\
(\mathrm{M}=\mathrm{NR}, \mathrm{F}=\mathrm{NR}) \\
\text { Mean age: } \mathrm{NR} \\
\text { Age range: } \mathrm{NR} \\
\text { Group I: } \\
\mathrm{N}=386 \\
(\mathrm{M}=303, \mathrm{~F}=83) \\
\text { Mean age: } 66 \\
\text { Age range: }<45-85+ \\
\rightarrow \text { Stable mechanical or } \\
\text { neurologic } \mathrm{OD} \\
\text { Group II: } \\
\mathrm{N}=40\end{array}$ & $\begin{array}{l}\text { HRQoL to OD: } \\
\text { SWAL-QOL (Swallowing Quality of Life } \\
\text { Questionnaire) } \\
\text { HRQoL: } \\
\text { NA } \\
\text { QoL: } \\
\text { MOS-items (Medical Outcomes Study) } \\
\text { FHS: } \\
\text { SWAL-CARE (Swallowing Quality of Care } \\
\text { Questionnaire) }\end{array}$ & $\begin{array}{l}\text { Group I: } \\
(\mathrm{N}=386 ; 91 \%) \\
\text { OD } \\
\text { Group II: } \\
(\mathrm{N}=40 ; 9 \%) \\
\text { No OD }\end{array}$ & $\begin{array}{l}\rightarrow \text { Group I reported significantly lower HRQoL (SWAL-QOL) and QoL } \\
\text { (MOS) compared with Group II }(p=0.000) \text {. }\end{array}$ \\
\hline
\end{tabular}




\begin{tabular}{|c|c|c|c|c|}
\hline Literature & $\begin{array}{l}\mathrm{N}, \text { Age in years/ Etiology of } \\
\text { OD }\end{array}$ & $\begin{array}{l}\text { HRQoL to OD, HRQoL, QoL, FHS, OD gold } \\
\text { standard }\end{array}$ & Treatment(s)/Groups & Key Finding(s)/Conclusion(s) \\
\hline & $\begin{array}{l}(\mathrm{M}=\mathrm{NR}, \mathrm{F}=\mathrm{NR}) \\
\text { Mean age: } 73 \\
\text { Age range: } 45-95 \\
\rightarrow \text { No OD (healthy controls) }\end{array}$ & $\begin{array}{l}\text { OD gold standard: } \\
\text { VFS } \\
\text { OD measure: } \\
\text { Patient report of food textures } \\
\text { Symptoms reported from SWAL-QOL }\end{array}$ & & \\
\hline $\begin{array}{l}\text { McHorney et al., } \\
2006 \text { [27] }\end{array}$ & $\begin{array}{l}\mathrm{N}=386 \\
(\mathrm{M}=303, \mathrm{~F}=83) \\
\text { Mean age: } 66 \\
\text { Age range: } 53-79 \\
\rightarrow \text { OD from cancer }(28 \%), \\
\text { vascular diseases }(16 \%), \\
\text { degenerative neurologic } \\
\text { diseases }(13 \%), \text { other } \\
\text { neurologic diseases }(9 \%), \\
\text { obstructive respiratory diseases } \\
(6 \%), \text { unknown } 12 \%), \text { trauma } \\
(4 \%), \text { chronic medical } \\
\text { conditions }(4 \%), \text { dementia } \\
(1 \%) \text {, other reasons }(7 \%) .\end{array}$ & $\begin{array}{l}\text { HRQoL to OD: } \\
\text { SWAL-QOL (Swallowing Quality of Life } \\
\text { Questionnaire) } \\
\text { HRQoL: } \\
\text { NA } \\
\text { QoL: } \\
\text { NA } \\
\text { FHS: } \\
\text { SWAL-CARE (Swallowing Quality of Care } \\
\text { Questionnaire) } \\
\text { OD gold standard: } \\
\text { VFS } \\
\text { OD measure: } \\
\text { PAS (Penetration Aspiration Scale or PENASP) } \\
\text { Oral transit duration (OTD) } \\
\text { Pharyngeal transit duration (PTD) } \\
\text { Total swallowing duration (TDS) }\end{array}$ & $\begin{array}{l}\text { All participants completed } \\
\text { both questionnaires. }\end{array}$ & $\begin{array}{l}\rightarrow \text { Correlations between OD measures (PAS, OTD, PTD and TDS) for } \\
\text { thin liquids and SWAL-QOL domains: } 11 \text { of the } 40 \text { correlations were } \\
\text { statistically significant }(r=-0.11 \text { to }-0.21 ; p<0.05 \text { ). Seven of the } \\
\text { correlations were between OTD and SWALQOL domains; } 1 \text { was between } \\
\text { TSD and SWALQOL domain "Communication"; } 3 \text { were between PAS } \\
\text { and SWALQOL domains "Food Selection", "Mental Health" and "Eating } \\
\text { Duration". } \\
\rightarrow \text { Correlations between OTD for thin liquids and SWALCARE found all } \\
3 \text { correlations statistically significant ( } r=-0.14 \text { to }-0.23 ; p<0.05 \text { ). No } \\
\text { significant correlations were found between PTD, TDS or PAS with } \\
\text { SWALCARE. } \\
\rightarrow \text { Correlations between OD measures (PAS, OTD, PTD and TDS) for } \\
\text { semisolids and SWAL-QOL domains: } 15 \text { of the } 40 \text { correlations were } \\
\text { statistically significant ( } r=-0.01 \text { to - } 0.29 ; p<0.05 \text { ). Six of the } \\
\text { correlations were between SWALQOL domains and OTD; } 2 \text { were } \\
\text { between PTD and SWALQOL domains "Social Functioning" and } \\
\text { "Communication", } 5 \text { were between TSD and SWALQOL domains; and } 2 \\
\text { were between PAS and SWALQOL domains "Food selection" and } \\
\text { "Mental Health". } \\
\rightarrow \text { Correlations between OD measures for thin liquids between OTD and } \\
\text { SWALCARE found all } 3 \text { of } 12 \text { correlations statistically significant }(r=- \\
0.14 \text { to - } 0.20 ; p<0.05 \text { ). These correlations were between OTD, TSD and } \\
\text { PAS. }\end{array}$ \\
\hline $\begin{array}{l}\text { Nguyen et al., } \\
2005[50]\end{array}$ & $\begin{array}{l}\mathrm{N}=104^{\mathrm{b}} \\
(\mathrm{M}=\mathrm{NR}, \mathrm{F}=\mathrm{NR}) \\
\text { Mean age: NR } \\
\text { Age range: NR } \\
\rightarrow \text { Participants after treatment } \\
\text { for HNC } \\
\text { Group I: } \\
\mathrm{N}=31 \\
(\mathrm{M}=30, \mathrm{~F}=1) \\
\text { Mean age: NR } \\
\text { Age range: } 46-75 \\
\text { Median: } 59 \\
\rightarrow \text { no or minimal OD (control) } \\
\text { Groups II-IV: } \\
\mathrm{N}=71 \\
(\mathrm{M}=71, \mathrm{~F}=0)\end{array}$ & $\begin{array}{l}\text { HRQoL to OD: } \\
\text { NA } \\
\text { HRQoL: } \\
\text { HADS (Hospital Anxiety and Depression Scale) } \\
\text { QoL: } \\
\text { UW-QOL (University of Washington Quality of Life } \\
\text { Questionnaire) } \\
\text { FHS: } \\
\text { NA } \\
\text { OD gold standard: } \\
\text { VFS or NR (Group I) } \\
\text { OD measure: }\end{array}$ & $\begin{array}{l}\text { Group I: } \\
\text { (N=31; 30\%) } \\
\text { No or minimal OD (Grade } 1 \\
\text { on SPS) } \\
\text { Group II: } \\
\text { (N=24; 23\%) } \\
\text { Mild OD (Grade 2-3 on SPS) } \\
\text { Group III: } \\
\text { (N=25; 24\%) } \\
\text { Moderate OD (Grade 4-5 on } \\
\text { SPS) } \\
\text { Group IV: } \\
\text { (N=24; 24\%) } \\
\text { Severe OD (Grade 6-7 on } \\
\text { SPS) }\end{array}$ & $\begin{array}{l}\rightarrow \text { Group II-IV (OD) versus Group I (no OD): statistically significant } \\
\text { lower HRQoL (HADS: HA and HD scores) and QoL (UW-QOL) }(p< \\
0.0005 \text { ) for patients with OD. } \\
\rightarrow \text { Group I-II combined versus Group III-IV combined: statistically } \\
\text { significant greater QoL (UW-QOL; } p<0.0005 \text { ) and HRQoL (HA and } \\
\text { HD; resp. } p<0.005 \text { and } p<0.0001 \text { ) in patients with no or mild } \\
\text { dysphagia. }\end{array}$ \\
\hline
\end{tabular}




\begin{tabular}{|c|c|c|c|c|}
\hline Literature & $\begin{array}{l}\mathrm{N}, \text { Age in years/ Etiology of } \\
\text { OD }\end{array}$ & $\begin{array}{l}\text { HRQoL to OD, HRQoL, QoL, FHS, OD gold } \\
\text { standard }\end{array}$ & Treatment(s)/ Groups & Key Finding(s)/Conclusion(s) \\
\hline & $\begin{array}{l}\text { Mean age: } \mathrm{NR} \\
\text { Age range: } \mathrm{NR} \\
\rightarrow \mathrm{OD}\end{array}$ & Swallowing Performance Scale (SPS) & $\begin{array}{l}\rightarrow \text { After undergoing } \\
\text { treatment for HNC and VFS, } \\
\text { all participants completed a } \\
\text { 7-point scale to stratify OD } \\
\text { severity and completed QoL } \\
\text { questionnaires. }\end{array}$ & \\
\hline $\begin{array}{l}\text { Nogueira et al., } \\
2015[51]\end{array}$ & $\begin{array}{l}\mathrm{N}=520 \\
(\mathrm{M}=145, \mathrm{~F}=372) \\
\text { Mean age: } 79 \\
\text { Age range: } 25-105 \\
\text { Included: } \\
\mathrm{N}=205 \\
(\mathrm{M}=57, \mathrm{~F}=146) \\
\text { Mean age: } 77 \\
\text { Age range: } 25-103 \\
\rightarrow \text { OD largely due to stroke } \\
\text { (20\%), dementia }(19 \%), \\
\text { psychosis }(7.3 \%) \text {, and } \\
\text { cardiomyopathy }(3.7 \%) \\
\\
\text { Group II } \\
\mathrm{N}=315 \\
\text { (M=89, } \mathrm{F}=226) \\
\text { Mean age: } 80 \\
\text { Age range: } 44-105 \\
\rightarrow \text { No OD }\end{array}$ & $\begin{array}{l}\text { HRQoL to OD: } \\
\text { HRQoL: } \\
\text { NA } \\
\text { QoL: } \\
\text { EQ-5D (EuroQol Group Portuguese Index) } \\
\text { FHS: } \\
\text { P-EAT-10 (Eating Assessment Tool, 10-item- } \\
\text { European Portuguese version) } \\
\text { OD gold standard: } \\
\text { NR } \\
\text { OD measure: } \\
\text { NR }\end{array}$ & $\begin{array}{l}\text { Group I: } \\
\text { (N=205; } 40 \%) \\
\text { OD } \\
\text { Group II: } \\
\text { (N=315; } 60 \% \\
\text { No OD }\end{array}$ & $\begin{array}{l}\rightarrow \text { Group I reported significantly lower EQ-5D scores compared with } \\
\text { Group II ( } p=0.014 \text { or less) for all domains except "Pain/Discomfort". }\end{array}$ \\
\hline $\begin{array}{l}\text { Pedersen et al., } \\
2016[52]\end{array}$ & $\begin{array}{l}\mathrm{N}=173 \\
(\mathrm{M}=138, \mathrm{~F}=35) \\
\text { Mean age: } 62 \\
\text { Age range: } 42-89 \\
\rightarrow \text { Participants with OD from } \\
\text { HNC undergoing } \\
\text { chemotherapy. }\end{array}$ & $\begin{array}{l}\text { HRQoL to OD: } \\
\text { MDADI (MD Anderson Dysphagia Inventory) } \\
\text { HRQoL: } \\
\text { NA } \\
\text { QoL: } \\
\text { NA } \\
\text { FHS: } \\
\text { NOD (Normalcy of Diet: sub-section of Performance } \\
\text { Status Scale or PSS) } \\
\text { OD gold standard: } \\
\text { FEES } \\
\text { OD measure: } \\
\text { PAS (Penetration Aspiration Scale) }\end{array}$ & $\begin{array}{l}\rightarrow \text { All participants completed } \\
\text { all questionnaires at } 3 \mathrm{~m} . \\
\text { post-treatment. }\end{array}$ & $\begin{array}{l}\rightarrow \text { Participants with penetration or aspiration (PAS) reported significantly } \\
\text { lower HRQoL (MDADI) compared to participants with no penetration or } \\
\text { aspiration }(p<0.001) \text {. } \\
\rightarrow \text { A strong correlation was reported between the MDADI and NOD }(r= \\
0.68,95 \% \text { CI }[0.59,0.76]) \text {, a weak correlation between the MDADI and } \\
\text { the PAS }(r=0.34,95 \% \text { CI }[0.19,0.47]) \text {, and a moderate correlation } \\
\text { between MDADI and WST: }(r=0.45,95 \% \text { CI }[0.32,0.57]) .\end{array}$ \\
\hline
\end{tabular}




\begin{tabular}{|c|c|c|c|c|}
\hline Literature & $\begin{array}{l}\mathbf{N}, \text { Age in years/ Etiology of } \\
\text { OD }\end{array}$ & $\begin{array}{l}\text { HRQoL to OD, HRQoL, QoL, FHS, OD gold } \\
\text { standard }\end{array}$ & Treatment(s)/Groups & Key Finding(s)/Conclusion(s) \\
\hline & & $\begin{array}{l}\text { NOD } \\
100 \text { ml Water Swallow Test (WST) }\end{array}$ & & \\
\hline $\begin{array}{l}\text { Pierce et al, } 2016 \\
\text { [53] }\end{array}$ & $\begin{array}{l}\mathrm{N}=101 \\
(\mathrm{M}=7, \mathrm{~F}=94) \\
\text { Mean: } 59 \\
\text { Age range: } 21-93 \\
\rightarrow \text { Participants with primary or } \\
\text { secondary Sjogren's syndrome, } \\
\text { including }(\mathrm{N}=65,64.4 \%) \text { who } \\
\text { classified themselves as having } \\
\text { a currently having OD. }\end{array}$ & $\begin{array}{l}\text { HRQoL to OD: } \\
\text { MDADI (MD Anderson Dysphagia Inventory) } \\
\text { HRQoL: } \\
\text { SF-36 (Short Form Health Survey) } \\
\text { QoL: } \\
\text { NA } \\
\text { FHS: } \\
\text { RSSPRI (Rheumatism Sjogren's Syndrome Patient } \\
\text { reported Index) } \\
\text { SSI (Sicca Symptoms Inventory) } \\
\text { OD gold standard: } \\
\text { None - self reported } \\
\text { OD measure: } \\
\text { Participant self-report }\end{array}$ & $\begin{array}{l}\rightarrow \text { All participants completed } \\
\text { all questionnaires. }\end{array}$ & $\begin{array}{l}\rightarrow \text { Participants who reported OD scored lower for HRQoL (MDADI) } \\
\text { compared with participants who did not report dysphagia }(p<0.0001) \text {. } \\
\rightarrow \text { Participants who reported OD scored significantly lower QoL on } 2 \text { of } \\
\text { the } 8 \text { SF-36 subscales: mental health }(p=0.042) \text { and general health }(p= \\
0.023) \text {. } \\
\rightarrow \text { Logistic modelling showed that } 6 \text { swallowing symptoms were } \\
\text { independently associated with the SF-36 subscales. These symptoms } \\
\text { seem to disproportionately contribute to reductions in specific aspects of } \\
\text { quality of life, including bodily pain and vitality. }\end{array}$ \\
\hline $\begin{array}{l}\text { Ramaekers et } \\
\text { al., } 2011[54]\end{array}$ & $\begin{array}{l}\mathrm{N}=396 \\
(\mathrm{M}=276, \mathrm{~F}=120) \\
\text { Mean age: } 63 \\
\text { Age range: } 20-99 \\
\rightarrow \text { HNC (at least } 6 \mathrm{~m} . \text { after } \\
\text { curative } \mathrm{RT} \text { alone or with } \\
\text { surgery and/or CT) }\end{array}$ & $\begin{array}{l}\text { HRQoL to OD: } \\
\text { NA } \\
\text { HRQoL: } \\
\text { NA } \\
\text { QoL: } \\
\text { EQ-5D (EuroQol-5D Questionnaire) } \\
\text { FHS: } \\
\text { NA } \\
\text { OD gold standard: } \\
\text { None - confirmed by clinical assessment } \\
\text { OD measure: } \\
\text { Subgroups were categorized by grades of xerostomia } \\
\text { and/or dysphagia (based on the Radiation Therapy } \\
\text { Oncology Group or RTOG scale) }\end{array}$ & $\begin{array}{l}\text { Group I: } \\
\text { (N=107; } 27 \% \text { ) } \\
\text { Xerostomia } \\
\text { Group II: } \\
\text { (N=28; } 7 \% \text { ) } \\
\text { OD } \\
\text { Group III: } \\
\text { (N=177; 45\%) } \\
\text { OD and xerostomia } \\
\text { Group IV: } \\
\text { (N=84; } 21 \% \text { ) } \\
\text { No OD/xerostomia } \\
\rightarrow \text { EQ-5D scores categorised } \\
\text { by presence/absence of } \\
\text { xerostomia and/or OD } \\
\text { severities. }\end{array}$ & $\begin{array}{l}\rightarrow \text { Groups with xerostomia and/or OD showed statistically significant } \\
\text { lower QoL (EQ-5D) compared to participants with no xerostomia or OD } \\
(p<0.022) \text {. OD influences HRQOL more so than xerostomia. }\end{array}$ \\
\hline $\begin{array}{l}\text { Starmer et al., } \\
2014[56]\end{array}$ & $\begin{array}{l}\mathrm{N}=111 \\
(\mathrm{M}=44, \mathrm{~F}=67) \\
\text { Mean age: } 51 \\
\text { Age range: } 20-81 \\
\rightarrow \text { Participants undergoing } \\
\text { CPA surgery, of which } 35 \%\end{array}$ & $\begin{array}{l}\text { HRQoL to OD: } \\
\text { MDADI (M.D. Anderson Dysphagia Inventory) } \\
\text { HRQoL: } \\
\text { NA } \\
\text { QoL: }\end{array}$ & $\begin{array}{l}\rightarrow \text { All participants completed } \\
\text { questionnaires immediately } \\
\text { post CPA surgery. } \\
\text { Comparisons performed } \\
\text { between postoperative } \\
\text { function and long-term }\end{array}$ & $\begin{array}{l}\rightarrow \text { Participants with OD compared to participants without OD reported } \\
\text { significantly lower QoL (MDADI; } p=0.021) \text { and FaCE scores }(p= \\
0.0001) \text {. }\end{array}$ \\
\hline
\end{tabular}




\begin{tabular}{|c|c|c|c|c|}
\hline Literature & $\begin{array}{l}\mathrm{N}, \text { Age in years/ Etiology of } \\
\text { OD }\end{array}$ & $\begin{array}{l}\text { HRQoL to OD, HRQoL, QoL, FHS, OD gold } \\
\text { standard }\end{array}$ & Treatment(s)/ Groups & Key Finding(s)/Conclusion(s) \\
\hline & $\begin{array}{l}\text { were assessed as having post- } \\
\text { operative OD }\end{array}$ & $\begin{array}{l}\text { NA } \\
\text { FHS: } \\
\text { VHI (Voice Handicap Index) } \\
\text { FaCE (Facial Clinimetric Evaluation) } \\
\text { OD gold standard: } \\
\text { FEES \& VFS - for some participants } \\
\text { OD measure: } \\
\text { Speech pathology assessment }\end{array}$ & $\begin{array}{l}\text { patient perception of } \\
\text { function. }\end{array}$ & \\
\hline $\begin{array}{l}\text { Tabor et al, } 2016 \\
\text { [57] }\end{array}$ & $\begin{array}{l}\mathrm{N}=82 \\
(\mathrm{M}=53, \mathrm{~F}=29) \\
\text { Mean age: } 62 \\
\text { Age range: NR } \\
\rightarrow \text { ALS } \\
\text { Group I: } \\
\mathrm{N}=45 \\
(\mathrm{M}=\mathrm{NR}, \mathrm{F}=\mathrm{NR}) \\
\text { Mean age: NR } \\
\text { Age range: NR } \\
\rightarrow \text { Participants with safe } \\
\text { swallow } \\
\text { Group II: } \\
\mathrm{N}=21 \\
\text { (M=NR, F=NR) } \\
\text { Mean age: NR } \\
\text { Age range: NR } \\
\rightarrow \text { Participants with penetration } \\
\text { Group III: } \\
\mathrm{N}=15 \\
\text { (M=NR, F=NR) } \\
\text { Mean age: NR } \\
\text { Age range: NR } \\
\rightarrow \text { Participants with aspiration } \\
\end{array}$ & $\begin{array}{l}\text { HRQoL to OD: } \\
\text { SWAL-QOL (Swallowing quality of life) } \\
\text { HRQoL: } \\
\text { NA } \\
\text { QoL: } \\
\text { ALSFRS-R (ALS functional rating scale-revised) } \\
\text { FHS: } \\
\text { NA } \\
\text { OD gold standard: } \\
\text { VFS } \\
\text { OD measure: } \\
\text { Participants divided into groups according to PAS } \\
\text { scores: ‘safe' swallowers (PAS } \leq 2 \text { ), people with } \\
\text { penetration (PAS 3-5), and with aspiration (PAS } \geq 6 \text { ) }\end{array}$ & $\begin{array}{l}\text { Group I: } \\
(\mathrm{N}=45 ; 55 \%) \\
\text { 'Safe' swallowers }(\mathrm{PAS}<2) \\
\text { Group II: } \\
(\mathrm{N}=21 ; 26 \%) \\
\text { 'Penetrators' (PAS: } 3-5) \\
\text { Group III: } \\
\text { 'Aspirators' (PAS >6). }(\mathrm{N}= \\
\text { 15; 19\%) } \\
\rightarrow \text { All participants completed } \\
\text { all questionnaires }\end{array}$ & $\begin{array}{l}\rightarrow \text { Significant negative correlation between SWAL-QOL (total scores) } \\
\text { and PAS score }(r=-0.39 ; p<0.001): \text { lower (worse) HRQoL (SWAL- } \\
\text { QOL total scores) associated with higher (worse) PAS scores. } \\
\rightarrow \text { Significant correlations between SWAL-QOL (total scores) and } \\
\text { ALSFRS-R }(r=0.23, p<0.05 \text { ), and SWAL-QOL (total scores) and } \\
\text { bulbar sub-scores of the ALSFRS-R ( } r=0.46, p<0.001 \text { ): higher (better) } \\
\text { HRQoL (SWAL-QOL total score) associated with higher (better) } \\
\text { ALSFRS-R scores. } \\
\rightarrow \text { Mean (SD) SWAL-QOL scores for Group I vs II vs III were } 81.2(2.3) \\
\text { vs } 77(3.4) \text { vs } 58.7 \text { (5.9), respectively, with a main effect observed } \\
\text { [F( } 2.78)=9.71, p<0.001] \text {. } \\
\rightarrow \text { Lower SWAL-QoL scores for group III vs Group I ( } p<0.001 \text { ), and } \\
\text { Group III vs Group II ( } p<0.001 \text { ). } \\
\rightarrow \text { All SWAL-QOL domains were significantly lower for Group III when } \\
\text { compared to Group I (p }<0.001 \text { ). Domains of "Fatigue" (p }<0.01 \text { ) and } \\
\text { "Symptoms" (p }<0.02) \text { were significantly worse in Group II when } \\
\text { compared to Group I. } \\
\rightarrow \text { When compared to Group II, all individual domains except "Eating } \\
\text { Duration", "Fatigue", "Fear" and "Food Selection" were significantly } \\
\text { lower for Group III (p }<0.003 \text { ) }\end{array}$ \\
\hline $\begin{array}{l}\text { Vanderwegen et } \\
\text { al., } 2012[58]\end{array}$ & $\begin{array}{l}\mathrm{N}=392 \\
(\mathrm{M}=240, \mathrm{~F}=152) \\
\text { Mean age: } \mathrm{NR} \\
\text { Age range: } \mathrm{NR} \\
\text { Group I: } \\
\mathrm{N}=268 \\
(\mathrm{M}=177, \mathrm{~F}=91) \\
\text { Mean age: } 68 \\
\text { Age range: } 20-81\end{array}$ & $\begin{array}{l}\text { HRQoL to OD: } \\
\text { DSWAL-QOL (Swallowing Quality-of-Life } \\
\text { Questionnaire - Dutch Version) } \\
\text { HRQoL: } \\
\text { SF-36 (Short-form Health Survey) } \\
\text { QoL: } \\
\text { NA }\end{array}$ & $\begin{array}{l}\text { Group I: } \\
(\mathrm{N}=268 ; 68 \%) \\
\text { OD } \\
\text { Group II: } \\
(\mathrm{N}=124 ; 32 \%) \\
\text { No OD }\end{array}$ & $\begin{array}{l}\rightarrow \text { A relationship between increased OD severities with decreased } \\
\text { HRQoL was found; participants with OD in Group I demonstrated } \\
\text { statistically significant }(p<0.000) \text { lower scores on all SWAL-QOL } \\
\text { subscales, when compared to those without OD in Group II. } \\
\rightarrow \text { The effect sizes are all large, with a mean of } 0.37 \text { and ranging from } \\
0.24 \text { ("Fatigue") to } 0.50 \text { ("Sleep"). The greatest differences were } \\
\text { observed for "Sleep", "Symptoms" and "General Burden". }\end{array}$ \\
\hline
\end{tabular}




\begin{tabular}{|c|c|c|c|c|}
\hline Literature & $\begin{array}{l}\mathrm{N}, \text { Age in years/ Etiology of } \\
\text { OD }\end{array}$ & $\begin{array}{l}\text { HRQoL to OD, HRQoL, QoL, FHS, OD gold } \\
\text { standard }\end{array}$ & Treatment(s)/ Groups & Key Finding(s)/Conclusion(s) \\
\hline & $\begin{array}{l}\rightarrow \text { Neurological or mechanical } \\
\text { OD due to HNC, Stroke, PD or } \\
\text { Zenker's diverticulum } \\
\text { Group II: } \\
\mathrm{N}=124 \\
(\mathrm{M}=63, \mathrm{~F}=61) \\
\text { Mean age: } 40 \\
\text { Age range: } 22-89 \\
\rightarrow \text { No OD (healthy controls) }\end{array}$ & $\begin{array}{l}\text { FHS: } \\
\text { NA } \\
\text { OD gold standard: } \\
\text { FEES or VFS } \\
\text { OD measure: } \\
\text { Diagnosed by a speech-language pathologist and/or } \\
\text { ENT surgeon }\end{array}$ & & \\
\hline
\end{tabular}

Note. Abbreviations (alphabetical order): ALS = Amyotrophic Lateral Sclerosis; CPA = Cerebello-Pontine Angle Surgery; NMD = Neuromuscular Disease; F = females; FEES = Fibre optic Endoscopic Evaluation of Swallowing; FEESST = Fibre optic Endoscopic Evaluation of Swallowing with Sensory Testing; FHS = Functional Health Status; HNC = Head and Neck Cancer; HRQoL = Health-Related Quality of Life; IPD = Idiopathic Parkinson's disease; M = males; $\mathrm{m}$. = month(s); NA= Not applicable to this study; NR = Information not reported; NPC = Nasopharyngeal Carcinoma; OD = Oropharyngeal Dysphagia; PD = Parkinson's disease; QoL = Quality of Life; RT = Radiotherapy; TBI = Traumatic Brain Injury; VFS = Videofluoroscopy; w. = week(s); yr = year(s). 
Table 4 Changes in health-related quality of life and quality of life in oropharyngeal dysphagia, and vice versa, after intervention

\begin{tabular}{|c|c|c|c|c|}
\hline Literature & $\mathrm{N}$, Age in years/ Etiology of OD & $\begin{array}{l}\text { HRQoL to OD, HRQoL, QoL, FHS, OD } \\
\text { gold standard }\end{array}$ & Treatment(s)/ Groups & Key Finding(s)/ Conclusion(s) \\
\hline $\begin{array}{l}\text { Argolo et al., } 2013 \\
{[35]}\end{array}$ & $\begin{array}{l}\mathrm{N}=15 \\
(\mathrm{M}=10, \mathrm{~F}=5) \\
\text { Mean age: } 59 \\
\text { Age range: } 50-68 \\
\rightarrow \text { PD with OD. } \\
\text { (H\&Y mean: } 2.23 \pm 0.7 \text {, disease } \\
\text { duration mean: } 79.3 \pm 46 \mathrm{~m} \text {.) }\end{array}$ & $\begin{array}{l}\text { HRQoL to OD: } \\
\text { DSWAL-QOL (Swallowing Quality of Life } \\
\text { Questionnaire - Dutch Version) } \\
\text { HRQoL: } \\
\text { NA } \\
\text { QoL: } \\
\text { NA } \\
\text { FHS: } \\
\text { NA } \\
\text { OD gold standard: } \\
\text { VFS } \\
\text { OD Measure: } \\
\text { Total Swallow Score (TTS; based on VFS) }\end{array}$ & $\begin{array}{l}\rightarrow \text { All participants completed } \\
\text { VFS and SWAL-QOL. } \\
\rightarrow \text { All participants underwent } \\
\text { oral motor exercises twice a } \\
\text { day, } 5 \text { days a w., for } 5 \mathrm{w} \text {. }\end{array}$ & $\begin{array}{l}\rightarrow \text { After intervention, } 9 / 15 \text { participants showed improved HRQoL } \\
\text { (DSWAL-QoL). The difference in this score before and after intervention } \\
\text { had no correlation with the difference in OD (TSS based on VFS): r= } \\
0.13(p=0.63) \text {. } \\
\rightarrow \text { Only the mean of difference in SWAL-QOL scores before and after } \\
\text { intervention for the "Fear" }(p=0.02) \text { and "Symptom frequency" }(p= \\
0.05) \text { domains were statistically significant. No other domains exhibited } \\
\text { statistically significant change. }\end{array}$ \\
\hline $\begin{array}{l}\text { Heijnen et al., } 2012 \\
{[43]}\end{array}$ & $\begin{array}{l}\mathrm{N}=85 \text { a } \\
\text { (M=65, F=23) } \\
\text { Mean age: } 68 \\
\text { Age range: } 42-81 \\
\rightarrow \text { IPD with OD } \\
\text { Group I: } \\
\mathrm{N}=28 \\
(\mathrm{M}=22, \mathrm{~F}=7) \\
\text { Mean age: } 69 \\
\text { Age range: } \mathrm{NR} \\
\text { Group IIa: } \\
\mathrm{N}=27 \\
\text { (M=20, F=9) } \\
\text { Mean age: } 65 \\
\text { Age range: } \mathrm{NR} \\
\text { Group IIb: } \\
\mathrm{N}=30 \\
\text { (M=23, F=9) } \\
\text { Mean age: } 66 \\
\text { Age range: } \mathrm{NR}\end{array}$ & $\begin{array}{l}\text { HRQoL to OD: } \\
\text { SWAL-QOL (Swallowing Quality of Life } \\
\text { Questionnaire) } \\
\text { MDADI (M.D. Anderson Dysphagia Inventory) } \\
\text { HRQoL: } \\
\text { NA } \\
\text { QoL: } \\
\text { NA } \\
\text { FHS: } \\
\text { DSS (Dysphagia Severity Scale) } \\
\text { FOIS (Functional Oral Intake Scale) } \\
\text { OD gold standard: } \\
\text { FEES \& VFS } \\
\text { OD Measure: } \\
\text { DSS (Dysphagia Severity Scale) } \\
\end{array}$ & $\begin{array}{l}\text { Group I: } \\
\text { (N=28; 33\%) } \\
\text { Traditional logopedic } \\
\text { dysphagia treatment (TT) } \\
\text { Group IIa: } \\
\text { (N=27; 32\%) } \\
\text { Neuromuscular electrical } \\
\text { stimulation - motor level } \\
\text { (NMES-M) } \\
\text { Group IIb: } \\
\text { (N=30; 35\%) } \\
\text { Neuromuscular electrical } \\
\text { stimulation - sensory level } \\
\text { (NMES-S) } \\
\rightarrow \text { Pre-, post- and follow-up } \\
\text { (after 3 m.) measurement } \\
\text { using SWAL-QOL, MDADI, } \\
\text { FOIS and DSS }\end{array}$ & $\begin{array}{l}\rightarrow \text { After treatment, all groups showed statistically significant } \\
\text { improvement on OD (DSS) and small positive effects on QoL (SWAL- } \\
\text { QOL and MDADI); no statistically significant differences were found } \\
\text { between the } 3 \text { treatment groups. } \\
\rightarrow \text { Significant therapy improvements were found for Group I on the total } \\
\text { MDADI score, the global assessment, and both the "Physical" and } \\
\text { "Emotional" subscales. } \\
\rightarrow \text { Significant therapy improvements were found for Group II on the } \\
\text { SWAL-QOL and MDADI total scores. } \\
\rightarrow \text { No significant correlations were found between QOL (SWAL-QOL } \\
\text { and MDADI, dietary intake (FOIS) or OD (DSS): } r<0.2 \text {. }\end{array}$ \\
\hline Lal et al., 2014 [44] & $\begin{array}{l}\mathrm{N}=20 \\
(\mathrm{M}=19, \mathrm{~F}=1) \\
\text { Mean age: } 59 \\
\text { Age range: } 38-75 \\
\end{array}$ & $\begin{array}{l}\text { HRQoL to OD: } \\
\text { None } \\
\text { HRQoL: }\end{array}$ & $\begin{array}{l}\rightarrow \text { All participants received } \\
\text { SIB-IMRT }\end{array}$ & $\begin{array}{l}\rightarrow \text { Pretreatment coughing and swallowing difficulties were greater in } \\
\text { Group I when compared to Group II, and also remained persistently } \\
\text { higher following treatment (although mean differences were not } \\
\text { statistically significant at } p=0.216 \text { and } 0.183 \text {, respectively) }\end{array}$ \\
\hline
\end{tabular}




\begin{tabular}{|c|c|c|c|c|}
\hline Literature & $\mathrm{N}$, Age in years/ Etiology of OD & $\begin{array}{l}\text { HRQoL to OD, HRQoL, QoL, FHS, OD } \\
\text { gold standard }\end{array}$ & Treatment(s)/ Groups & Key Finding(s)/ Conclusion(s) \\
\hline & $\begin{array}{l}\rightarrow \text { Patients with OD due to HNC } \\
\text { Group I: } \\
\mathrm{N}=7 \\
(2 / 7 \dagger \text { within } 1 \mathrm{~m} \text {. from baseline; } \\
1 / 7 \dagger \text { date unknown) } \\
\text { (M=NR, F=NR) } \\
\text { Mean age: NR } \\
\text { Age range: NR } \\
\rightarrow \text { Aspirators (OD confirmed) } \\
\text { Group II: } \\
\mathrm{N}=13 \\
(1 / 13 \dagger \text { within } 3 \mathrm{~m} . \text { from baseline) } \\
(\mathrm{M}=\mathrm{NR}, \mathrm{F}=\mathrm{NR}) \\
\text { Mean age: NR } \\
\text { Age range: } \mathrm{NR} \\
\rightarrow \text { Non-aspirators (OD not } \\
\text { confirmed) }\end{array}$ & $\begin{array}{l}\text { EORTC-QLQ-C30 (European Organisation for } \\
\text { Research and Treatment of Cancer core } \\
\text { questionnaire) } \\
\text { EORTC-QLQ H\&N35 C30 (European } \\
\text { Organisation for Research and Treatment of } \\
\text { Cancer head \& neck questionnaire) } \\
\text { QoL: } \\
\text { NA } \\
\text { FHS: } \\
\text { NA } \\
\text { OD gold standard: } \\
\text { VFS }\end{array}$ & $\begin{array}{l}\rightarrow \text { All participants completed } \\
\text { all questionnaires pre- and } \\
\text { post- }(3 \text { and } 6 \mathrm{~m} \text {.) treatment. }\end{array}$ & $\begin{array}{l}\rightarrow \text { No significant difference was found between Group I and Group II in } \\
\text { terms of general QoL by the QLQ-C } 30 \text { at baseline and } 3 \mathrm{~m} \text {. post- } \\
\text { treatment. } \\
\rightarrow \text { Mean QoL scores (baseline and } 3 \mathrm{~m} \text {.) show improved HRQoL scores } \\
\text { in both groups after intervention, however, no statistical analyses } \\
\text { performed. }\end{array}$ \\
\hline Lin et al., $2011[47]$ & $\begin{array}{l}\mathrm{N}=20 \\
(\mathrm{M}=12, \mathrm{~F}=8) \\
\rightarrow \text { NPC with OD } \\
\text { Group I: } \\
\mathrm{N}=10 \\
(\mathrm{M}=6, \mathrm{~F}=4) \\
\text { Mean age: } 52 \\
\text { Age range: } 37-64 \\
\rightarrow \text { FES group } \\
\text { Group II: } \\
\mathrm{N}=10 \\
\text { (M=6, F=4) } \\
\text { Mean age: } 56 \\
\text { Age range: } 40-72 \\
\rightarrow \text { HRP group }\end{array}$ & $\begin{array}{l}\text { HRQoL to OD: } \\
\text { MDADI (M.D. Anderson Dysphagia Inventory) } \\
\text { HRQoL: } \\
\text { NA } \\
\text { QoL: } \\
\text { NA } \\
\text { FHS: } \\
\text { NA } \\
\text { OD gold standard: } \\
\text { VFS } \\
\text { OD Measure: } \\
\text { PAS (Penetration Aspiration Scale) and } \\
\text { temporal/spatial variables (based on VFS) } \\
\end{array}$ & $\begin{array}{l}\text { Group I: } \\
(\mathrm{N}=10 ; 50 \%) \\
\text { Functional electrical } \\
\text { stimulation (FES) over } 15 \\
\text { sessions } \\
\text { Group II: } \\
\text { (N=10; 50\%) } \\
\text { Home rehabilitation program } \\
\text { (HRP) of self-swallowing } \\
\text { exercises } \\
\\
\rightarrow \text { All participants completed } \\
\text { MDADI and VFS before and } \\
\text { after treatment. }\end{array}$ & $\begin{array}{l}\rightarrow \text { When comparing post- and pretreatment, Group I showed significantly } \\
\text { higher HRQoL (MDADI) ( } p=0.003) \text {, whereas Group II displayed no } \\
\text { significance change; comparison of group differences between pre- and } \\
\text { post-treatment (MDADI) were not significant. } \\
\rightarrow \text { Group I showed significantly more improvement than group II in the } \\
\text { PAS and velocity of the hyoid bone, but no significant difference was } \\
\text { found in hyoid displacement. }\end{array}$ \\
\hline $\begin{array}{l}\text { Rogus-Pulia et al., } \\
2016[55]\end{array}$ & $\begin{array}{l}\mathrm{N}=34 \\
(\mathrm{M}=33, \mathrm{~F}=1) \\
\text { Mean age: } 70 \\
\text { Age range: } 41-96 \\
\rightarrow \text { Patients with OD due to HNC } \\
(38 \%) \text {, respiratory (29\%), } \\
\text { neurologic (12\%), } \\
\text { gastroesophageal (9\%), dementia } \\
(6 \%) \text {, connective tissue (3\%) and } \\
\text { cardiac (3\%) }\end{array}$ & $\begin{array}{l}\text { HRQoL to OD: } \\
\text { SWAL-QOL (Swallowing Quality of Life } \\
\text { Questionnaire - Urdu-translated version) } \\
\text { HRQoL: } \\
\text { NA } \\
\text { QoL: } \\
\text { NA }\end{array}$ & $\begin{array}{l}\rightarrow \text { All participants underwent } \\
\text { Device-Facilitated Isometric } \\
\text { Progressive Resistance } \\
\text { Oropharyngeal (D-F IPRO) } \\
\text { therapy for } 8 \mathrm{w} \text {. } \\
\rightarrow \text { All participants completed } \\
\text { SWAL-QOL and VFS pre- } \\
\text { and post- (after } 4 \text { and } 8 \mathrm{w} .) \\
\text { measurement. }\end{array}$ & $\begin{array}{l}\rightarrow \text { Measures of aspiration (PAS) and measures of oropharyngeal residue } \\
\text { did not change significantly after } 8 \text { w. } \\
\rightarrow \text { HRQoL (SWAL-QOL) improved significantly on } 8 \text { of } 11 \text { subscales of } \\
\text { the SWAL-QOL: "Eating Desire" (effect estimate }=19.5, p<0.001 \text { ), } \\
\text { "Physical" (effect estimate }=11, p=0.003 \text { ), "Food" (effect estimate }= \\
11.4, p=0.04 \text { ), "Communication" (effect estimate }=6.5, p=0.03 \text { ), } \\
\text { "Fear" (effect estimate }=10.5, p=0.2) \text {, "Mental Health" (effect estimate } \\
=19, p<0.001) \text {, "Social" (effect estimate }=11.3, p=0.02 \text { ), and } \\
\text { "Fatigue" (effect estimate }=13.3, p<0.001 \text { ). }\end{array}$ \\
\hline
\end{tabular}




\begin{tabular}{|c|c|c|c|c|}
\hline Literature & $\mathrm{N}$, Age in years/ Etiology of OD & $\begin{array}{l}\text { HRQoL to OD, HRQoL, QoL, FHS, OD } \\
\text { gold standard }\end{array}$ & Treatment(s)/Groups & Key Finding(s)/ Conclusion(s) \\
\hline & & $\begin{array}{l}\text { FHS: } \\
\text { FOIS (Functional Oral Intake Scale) } \\
\text { VAS (swallowing-related effort) } \\
\text { OD gold standard: } \\
\text { VFS } \\
\text { OD Measure: } \\
\text { PAS and oropharyngeal residue measures (based } \\
\text { on VFS) }\end{array}$ & & $\begin{array}{l}\rightarrow \text { Significant improvements in FHS (FOIS) also occurred between pre } \\
\text { and post measures (effect estimates }=0.4, p=0.02 \text { ). }\end{array}$ \\
\hline $\begin{array}{l}\text { Zhang et al, } 2014 \\
{[59]}\end{array}$ & $\begin{array}{l}\mathrm{N}=58 \\
(\mathrm{M}=43, \mathrm{~F}=15) \\
\text { Mean age: NR; median: } 56.6 \\
\text { Age range: } 45-81 \\
\rightarrow \text { Tongue cancer with surgery } \\
\text { (i.e. partial tongue resection) and } \\
\text { OD }\end{array}$ & $\begin{array}{l}\text { HRQoL to OD: } \\
\text { NA } \\
\text { HRQoL: } \\
\text { NA } \\
\text { QoL: } \\
\text { Zung SDS (Zung Self-rating Depression Scale) } \\
\text { FHS: } \\
\text { WST (Water Swallow Test) } \\
\text { OD gold standard: } \\
\text { None } \\
\text { OD measure: } \\
\text { WST }\end{array}$ & $\begin{array}{l}\rightarrow \text { Treatment protocol } \\
\text { including direct (diet } \\
\text { modification, environmental } \\
\text { arrangements, positioning } \\
\text { and swallowing strategies) } \\
\text { and indirect (lip and lingual } \\
\text { exercises, thermal } \\
\text { stimulation) therapies } \\
\rightarrow \text { Pre-post assessment after } \\
10 \text { days of dysphagia } \\
\text { treatment using WST and } \\
\text { Zung SDS }\end{array}$ & $\begin{array}{l}\rightarrow \text { Significant improvements in swallow function (WST; } p=0.027 \text { ) and } \\
\text { depression (Zung SDS; } p=0.034 \text { ) after treatment. } \\
\rightarrow \text { Overall, patients with less than 50\% tongue resection had lower pre- } \\
\text { post test scores on WST and Zung SDS than patients with greater than } \\
50 \% \text { resection. (Higher scores indicate at higher level of dysfunction.) } \\
\rightarrow \text { Also, patients with early stage tumors had lower pre-post test scores } \\
\text { on WST and Zung SDS than patients with advanced tumors. } \\
\rightarrow \text { Lower swallow function scores (WST) were associated with lower } \\
\text { depression scores (Zung SDS), however this was not examined } \\
\text { statistically }\end{array}$ \\
\hline $\begin{array}{l}\text { Zhang et al, } 2016 \\
\text { [60] }\end{array}$ & $\begin{array}{l}\mathrm{N}=82 \\
(\mathrm{M}=52, \mathrm{~F}=30) \\
\text { Mean age: } 62 \\
\text { Age Range: NR } \\
\rightarrow \text { Medullary (brainstem) } \\
\text { infarction with OD } \\
\text { Group I: } \\
\mathrm{N}=27 \\
(\mathrm{M}=17, \mathrm{~F}=10) \\
\text { Mean age: } 62(\mathrm{SD}=8.7) \\
\text { Age range: } \mathrm{NR} \\
\text { Group II: } \\
\mathrm{N}=28 \\
\text { (M=16, F=12) } \\
\text { Mean age: } 61.3(\mathrm{SD}=7.1) \\
\text { Age range: } \mathrm{NR} \\
\text { Group III: } \\
\mathrm{N}=27\end{array}$ & $\begin{array}{l}\text { HRQoL to OD: } \\
\text { SWAL-QOL (Swallowing Quality of Life } \\
\text { Questionnaire) } \\
\text { HRQoL: } \\
\text { NA } \\
\text { QoL: } \\
\text { NA } \\
\text { FHS: } \\
\text { FOIS (Functional Oral Intake Scale) } \\
\text { WST (Water Swallow Test) } \\
\text { SSA (Standardised swallowing assessment) } \\
\text { OD gold standard: } \\
\text { VFS } \\
\text { OD measure: }\end{array}$ & $\begin{array}{l}\text { Group I: } \\
\text { Traditional Treatment } \\
\text { Group II: } \\
\text { Traditional Treatment plus } \\
\text { sensory treatment } \\
\text { (vocaSTIM-Master) } \\
\text { Group III: } \\
\text { Traditional Treatment plus } \\
\text { motor treatment } \\
\text { (multifunctional nerve } \\
\text { rehabilitation system) } \\
\rightarrow \text { Pre-post assessments } \\
\text { (after 4 w.) measurement } \\
\text { using WST, SSA, FOIS and } \\
\text { SWAL-QOL }\end{array}$ & $\begin{array}{l}\rightarrow \text { Significant improvements in swallow function (WST and SSA) and } \\
\text { QoL (SWAL-QOL) were identified after treatment }(p \leq 0.01) \text {. Group II } \\
\text { experienced significantly greater gains across all measures in comparison } \\
\text { to group III. Both groups II and III experienced greater gains across all } \\
\text { measures compared with Group I. }\end{array}$ \\
\hline
\end{tabular}




\begin{tabular}{|c|c|c|c|c|}
\hline Literature & $\mathrm{N}$, Age in years/ Etiology of OD & $\begin{array}{l}\text { HRQoL to OD, HRQoL, QoL, FHS, OD } \\
\text { gold standard }\end{array}$ & Treatment(s)/ Groups & Key Finding(s)/ Conclusion(s) \\
\hline & $\begin{array}{l}(\mathrm{M}=19, \mathrm{~F}=8) \\
\text { Mean age: } 62.2(\mathrm{SD}=9.2) \\
\text { Age range: } \mathrm{NR}\end{array}$ & $\begin{array}{l}\text { FOIS } \\
\text { WST } \\
\text { SSA }\end{array}$ & & \\
\hline $\begin{array}{l}\text { Zheng et al., } 2014 \\
\text { [34] }\end{array}$ & $\begin{array}{l}\mathrm{N}=89 \\
(\mathrm{M}=\mathrm{NR}, \mathrm{F}=\mathrm{NR}) \\
\text { Mean age: NR } \\
\text { Age range: } 53-79 \\
\rightarrow \text { Patients with OD due to SLC } \\
\text { undergoing horizontal partial } \\
\text { laryngectomy surgery } \\
\text { Group I: } \\
\mathrm{N}=45 \\
(\mathrm{M}=30, \mathrm{~F}=15) \\
\text { Mean age: NR } \\
\text { Age range: NR } \\
\rightarrow \text { Patient to patient education, } \\
\text { from pts who had previously } \\
\text { experienced the surgery, } \\
\text { Group II: } \\
\mathrm{N}=44 \\
\text { (M=24, F=20) } \\
\text { Mean age: NR } \\
\text { Age range: NR } \\
\rightarrow \text { Control group; nil patient to } \\
\text { patient education }\end{array}$ & $\begin{array}{l}\text { HRQoL to OD: } \\
\text { SWAL-QOL (Swallowing Quality of Life } \\
\text { Questionnaire) } \\
\text { HRQoL: } \\
\text { QLQ-C30 (EORTC Core Questionnaire) } \\
\text { QoL: } \\
\text { FHS: } \\
\text { VAS (swallowing ability) } \\
\text { OD gold standard: } \\
\text { None - NR (clinical or self-assessment) } \\
\text { OD Measure: } \\
\text { VAS (swallowing ability) }\end{array}$ & $\begin{array}{l}\text { Group I: } \\
\mathrm{N}=45 \\
\text { Received "Patient to patient } \\
\text { education" (from people who } \\
\text { had previously experienced } \\
\text { the surgery) } \\
\text { Group II: } \\
\mathrm{N}=44 \\
\text { (M=24, F=20) } \\
\text { No "Patient-to Patient } \\
\text { Education": i.e. control } \\
\rightarrow \text { All participants completed } \\
\text { all questionnaires before } \\
\text { surgery and at } 0.5,1,3 \text {, and } 6 \\
\text { m. post-surgery. }\end{array}$ & $\begin{array}{l}\rightarrow \text { One w. after eating (most patients were allowed to eat at } 0.5 \mathrm{~m} \text {. post } \\
\text { surgery), FHS (VAS) for Group I improved significantly }(p<0.05 \text { ); with } \\
\text { no significant change for Group II. } \\
\rightarrow \text { At } 0.5 \text { and } 1 \mathrm{~m} \text {. after eating, QoL scores (SWAL-QoL and EORTC } \\
\text { QLQ-C30) improved significantly ( } p<0.05 \text { ) for Group I; no obvious } \\
\text { change in Group II. } \\
\rightarrow \text { Global QoL scores were lowest at } 0.5 \mathrm{~m} \text {. post treatment and had } \\
\text { positive correlations with swallowing problems in both groups. }\end{array}$ \\
\hline
\end{tabular}

Note. Abbreviations (alphabetical order): F = females; FEES = Fibre optic Endoscopic Evaluation of Swallowing; FHS = Functional Health Status; HNC = Head and Neck Cancer; HRQoL = Health-Related Quality of Life; H\&Y = Hoehn and Yahr scale; IPD = Idiopathic Parkinson's disease; M = males; $\mathrm{m}$. = month(s); NA= Not applicable to this study; NR = Information not reported; NPC = Nasopharyngeal Carcinoma; OD = Oropharyngeal Dysphagia; PD = Parkinson's disease; QoL = Quality of Life; SIB-

IMRT $=$ Simultaneous Integrated boost technique of Intensity - Modulated Radiotherapy; SLC $=$ Supraglottic Laryngeal Cancer; VAS = Visual Analogue Scale; VFS =

Videofluoroscopy; w. = week(s). 


\section{Discussion}

\section{Caveats in research}

The purpose of this study was to investigate the relationships between HRQoL and OD and to report on changes in HRQoL and OD following intervention. Thirty-five studies met the eligibility criteria of which 27 studies reported on the relationships between HRQoL and OD, and eight studies reported on changes in HRQoL and OD following intervention. Although the prevalent theme emerging from the literature was that the presence of OD decreases HRQoL and vice versa, and a decrease in OD severity may relate to improved HRQoL, this interpretation has several caveats.

Relatively few studies were published considering the detrimental impact OD can have on individuals' HRQoL. Particularly studies incorporating HRQoL questionnaires both preand post-intervention (Table 4) are scarce. Furthermore, the hierarchical level of evidence of the included articles was restricted; the lack of randomized controlled trials may limit the strength of findings of this review.

The differing terms for dysphagia found in literature can make data ambiguous. In many of the included articles, it is unclear whether the term dysphagia is defined as oropharyngeal or esophageal dysphagia, or more broadly as swallowing difficulties. Some studies use definitions of OD based on visuo-perceptual interpretations of gold standard recordings (VFS or FEES), such as the presence of aspiration on predefined bolus consistencies (e.g., Campbell, Spinelli [38] and Tabor, Gaziano [57]). Other authors provide additional details on patients' dysphagic complaints and characteristics; for example, Heijnen, Speyer [43] describe problems of bolus forming, slow eating, oropharyngeal passage disorder, coughing while drinking, abnormal amounts of residue, and severe aspiration. Conversely many other studies utilised a very imprecise definition of OD, such as 
'broad range of swallowing problems from a variety of medical diagnoses' [36] or 'posttreatment subjective dysphagia' in HNC patients [39].

\section{HRQoL and etiology of OD}

As relationships between increased OD severity and decreased HRQoL are repeatedly identified, it begs the question as to what the main contributing factor is for reduced HRQoL. Is patients' decreased HRQoL only associated with increased severity of OD, or is it mediated or moderated by general factors of underlying diseases? For example, Leow, Huckabee [46] reported statistically significant differences on SWAL-QOL subscales for participants with OD and PD, when compared to those without [46]. Whether deemed a confounding factor or the ultimate result of increased OD severities, the HRQoL of participants with late stage PD appeared to concomitantly decrease alongside their disease progression [46]. They presented with statistically significant lower scores when compared to their early stage counterparts. This is in line with Tabor, Gaziano [57] reporting reduced HRQoL alongside ALS disease progression. No study was retrieved from the literature, conducting a multivariate regression analysis to concurrently account for the effect of OD severity in the improvement of HRQoL along with other confounding variables that may influence HRQoL, such as the impact of underlying diseases.

\section{Methodology and data analysis}

Many participants of the included studies in this review consisted of patients with HNC $(43 \%)$ or neurological diseases $(14 \%)$. Some of the studies $(31 \%)$ combined both oncological and neurological patients within the same study design. Furthermore, HRQoL subgroups were stratified by patient characteristics of OD severity (or healthy controls), treatment, comorbidities or underlying disease stage. Most studies describe comparisons between groups presenting with different degrees of OD severity as defined by a variety of measures and variables based on gold standards, clinical assessments, or patients' self-evaluation. 
Whereas most studies support their results by statistical findings, some authors only present descriptive data (e.g., group mean data) [26, 37, 44, 45]. Furthermore, if reporting on the statistical significance of relationships between HRQoL and severity of OD, authors do not always provide details on non-significant results. Problems may arise if only significant results are presented, thus introducing reporting bias. Related issues may arise from the number of statistical analyses performed; for example, Florie, Baijens [42] found statistically significant mean differences on a HRQoL scale between HNC patients categorised according to severity levels as determined by FEES; however, only three out of 60 analyses indicated that worse swallowing functioning was associated with lower HRQoL, whereas no significant results were found for the remaining 57 analyses. Although the authors did refer to the nonsignificant results, no adjustment for multiple comparisons was used, thus increasing the risk for Type 1 errors.

Another issue refers to the lack of quantifying the magnitude of the identified significant associations between HRQoL and OD severity. Twenty percent of the included studies (7 articles) presented limited information on correlation coefficients.

Aggregated data on the level of evidence of the included studies identified most articles classified at NHMRC level IV (89\%) or case series. The remaining four articles were classified at NHMRC level III-1 (pseudo-RCTs). Overall, the level of evidence of the included articles was rather low and longitudinal study designs were very scarce. Additionally, even though studies having less than 15 participants with OD were excluded from this review and the mean total number of participants for all 35 studies was 151 (SD 161), numbers per subgroup could be much lower, thus limiting the generalizability of outcomes and increasing the likelihood that the studies were underpowered, thus increasing the chances of Type 2 errors. As such, small subgroup populations may have very likely led 
to non-significant relations between HRQoL and severity of OD (e.g., Argolo, Sampaio [35] and Lin, Hsiao [47]).

This review included data from studies that may not necessarily have been designed to support our study purposes. For example, some studies reported on the validation of a HRQoL measure (e.g., Asadollahpour, Baghban [36]; Bogaardt, Speyer [26]; Carlsson, Ryden [39]) whereas other studies were designed to retrieve prevalence data on OD (e.g., García-Peris, Paron [18]). This review only selected data that supported our study purpose.

\section{Assessments}

For the purpose of generalization, it is integral for the assessments used to quantify OD severity to be conducted uniformly. However, both clinical protocols as well as the combination of assessments implemented to measure patients' severity of OD, varied greatly. Furthermore, the use of VFS or FEES (gold standard assessments) did not resolve the lack of standardisation in, for example, number of swallow trials, viscosities or bolus volumes, or measures for visuo-perceptual interpretation of recordings of swallowing. Even so, when evaluating dysphagia as a disease symptom, physiological measures of airway protection including VFS and FEES, are important to use in combination with self-report measures representing the patient's perspective [7].

Choices in the selection of assessments within clinical environments to quantify OD severity and to measure the impact of OD on HRQoL, may be influenced by factors such as the availability of VFS or FEES, time constraints during clinics or clinicians' personal preferences. Still, the choice of using of an assessment can only be justified if the psychometric properties of that particular assessment are robust. In this review, the psychometric properties of some included questionnaires are not well established. Future research may establish the quality of psychometric data; however, using questionnaires with 
known poor properties should be avoided as outcome data cannot be interpreted correctly [28].

\section{Limitations}

In relation to the limitations of this current review, only English publications were included, thus excluding possible results from non-English studies. Further, esophageal dysphagia was excluded, whereas it may also be important to study the differences in HRQoL for patients with OD and those experiencing esophageal dysphagia. Finally, authors of included studies were not contacted directly, so information may have been overlooked.

\section{Conclusion and Recommendations for Future Research}

In conclusion, based on the findings of this review an inverse bidirectional relationship between decreased HRQoL and increased OD severity was established. Following intervention, changes were evident through improved HRQoL with decreased OD severity. As swallowing difficulties have a major effect on the HRQoL of a patient, clinicians should be aware of and consider the potential negative impact of OD onto HRQoL $[1,10]$. This should be considered by including dysphagia-specific QoL measures as part of the assessment of OD. As limited information is available it seems apparent that more research of adequate quality is needed, investigating the relationships between a person's OD and overall QoL.

The following recommendations are made for future research: 1) more research investigating the relationships between HRQoL and OD is needed; 2) future studies should, where appropriate, implement a randomized controlled trial study design to formulate stronger evidence; 3) future studies examining the relationships between HRQoL and OD should quantify the severity of OD, severity of underlying diseases and both general QoL and HRQoL using well validated measures; 4) future studies should consider conducting multivariate regression analysis to simultaneously account for the impact of OD severity in 
the improvement of HR-QoL alongside other confounding variables that may influence HRQoL; 5) future studies need less ambiguous terminology referring to the type of dysphagia in order to allow more effective comparison of research; and 6) gold standard assessment protocols should be standardized to allow more effective comparison of research.

\section{Acknowledgements}

The authors declare they have no conflict of interest.

We would like to thank the librarians of Waleus Library (Leiden University Medical Center, the Netherlands) for formulating the literature search strategies. 


\section{References}

1. Cichero, J.A. and K.W. Altman, Definition, prevalence and burden of oropharyngeal dysphagia: A serious problem among older adults worldwide and the impact on prognosis and hospital resources. Nestle Nutrition Institute Workshop Service. Nestic Ltd., 2012(72): p. 1-11.

2. Martino, R., G. Pron, and N. Diamant, Screening for oropharyngeal dysphagia in stroke: Insufficient evidence for guidelines. Dysphagia, 2000. 5(1): p. 19-30.

3. García, J.M. and E. Chambers, Managing dysphagia through diet modifications: Evidence-based help for patients with impaired swallowing. AJN, 2010. 110(11): p. 26-33.

4. Rofes, L., Arreola, V., Almirall, J., Cabre, M., Campins, L., García-Peris, P., Speyer, R., \& Clave, P. , Diagnosis and management of oropharyngeal dysphagia and its nutritional and respiratory complications in the elderly. Gastroenterol Res Pract, 2011.

5. Carneiro, D., et al., Quality of life related to swallowing in Parkinson's disease. Dysphagia, 2014. 29(5): p. 578-82.

6. da Silva Brandão, D.M., J.L. da Silva Nascinmento, and L.G. Vianna, Functional capacity and quality of life among elderly patients with or without OD after an ischemic stroke. Rev Assoc Med Bras, 2010. 56(6): p. 738-743.

7. Finizia, C., et al., A cross-sectional validation study of the Swedish version of SWALQOL. Dysphagia, 2012. 27(3): p. 325-35.

8. Pinchot, S.N., Youngwirth, L., Rajamanickam, V., Schaefer, S., Sippel, R., Chen, H., Changes in swallowing-related quality of life after parathyroidectomy for hyperparathyroidism: a prospective cohort study. Oncologist, 2012. 17(10): p. 12716.

9. Swan, K., et al., Living with oropharyngeal dysphagia: Effects of bolus modification on health-related quality of life - A systematic review. Qual Life Res, 2015. 24(10): p. 2447-2456.

10. Cichero, J.A., et al., The need for international terminology and definitions for texture-modified foods and thickened liquids used in dysphagia management: Foundations of a global initiative. Curr Phys Med Rehabil Rep, 2013(1): p. 280-291.

11. Marik, P.E., \& Kaplan, D. , Aspiration pneumonia and dysphagia in the elderly. Chest, 2003(124): p. 328-336.

12. Kertscher, B., et al., Prevalence of oropharyngeal dysphagia in the Netherlands: $A$ telephone survey. Dysphagia, 2015. 30(2): p. 114-120.

13. European Society for Swallowing Disorders (ESSD). Position statement on the standardization of diagnostic methods - videofluoroscopy (VFS) and fiberoptic endoscopic evaluation of swallowing (FEES). 2nd ESSD Congress 2012; Available from: www.myessd.org.

14. Eslick, G. and N. Talley, Dysphagia: Epidemiology, risk factors and impact on quality of life - a population-based study. Alimentary Pharmacology and Therapeutics, 2008. 27(10): p. 971-979.

15. Roden, D.F. and K.W. Altman, Causes of dysphagia among different age groups $-A$ systematic review of the literature. Otolaryngol Clin N Am, 2013. 46(6): p. 965-987. 
16. Airoldi, M., et al., Functional and psychological evaluation after flap reconstruction plus radiotherapy in oral cancer. Head \& Neck, 2011. 33(4): p. 458-468.

17. Rodrigues, B., et al., Silent saliva aspiration in Parkinson's disease. Mov Disord, 2011. 26(1): p. 138-141.

18. García-Peris, P., et al., Long-term prevalence of oropharyngeal dysphagia in head and neck cancer patients: Impact on quality of life. Clinical Nutrition, 2007. 26(6): p. 710717.

19. Maclean, J., S. Cotton, and A. Perry, Dysphagia following a total laryngectomy: The effect on quality of life, functioning, and psychological well-being. Dysphagia, 2009. 24(3): p. 314-321.

20. Martino, R., Foley, N., Bhogal, S., Diamant, N., Speechley, M., \& Teasell, R. , Dysphagia after stroke. Stroke, 2005. 36(12): p. 2756-2763.

21. Asadi-Lari, M., Tamburini, M., \& Gray, D. , Patients' needs, satisfaction, and health related quality of life: Towards a comprehensive model. Health Qual Life Outcomes 2004. 2(1): p. 32-46.

22. World Health Organisation. WHOQOL: Measuring quality of life. 1997 [cited 2015 25th March]; Available from: http://www.who.int/mental_health/media/68.pdf.

23. Speyer, R., et al., Quality of life in oncological patients with oropharyngeal dysphagia: Validity and reliability of the dutch version of the MD anderson dysphagia inventory and the deglutition handicap index. Dysphagia, 2011. 26(4): p. 407-414.

24. Ferrans, C.E., Zerwic, J. J., Wilbur, J. E., \& Larson, J. L. , Conceptual model of healthrelated quality of life. JNS, 2005. 37(4): p. 336-342.

25. American Thoracic Society. Functional status. 2007; Available from: www.qol.thoracic.org.

26. Bogaardt, H.C.A., et al., Cross-cultural adaption and validation of the dutch version of SWAL-QoL. Dysphagia, 2009. 24(1): p. 66-70.

27. McHorney, C.A., et al., Clinical validity of the SWAL-QOL and SWAL-CARE outcome tools with respect to bolus flow measures. Dysphagia, 2006. 21(3): p. 141-148.

28. Timmerman, A.A., et al., Psychometric characteristics of health-related quality-of-life questionnaires in oropharyngeal dysphagia. Dysphagia, 2014. 29(2): p. 183-98.

29. Speyer, R., Oropharyngeal dysphagia: Screening and assessment. Otolaryngologic Clinics of North America, 2013. 46(6): p. 989-1008.

30. Moher, D., Liberati, A., Tetzlaff, J., Altman, D. G., \& The PRISMA Group, Preferred reporting items for systematic reviews and meta-analyses: The PRISMA statement. PLoS Medicine, 2009. 6(7): p. 1-6.

31. Kmet, L.M., R.C. Lee, and L.S. Cook, Standard quality assessment criteria for evaluating primary research papers from a variety of fields. 2004, Edmonton, University of Calgary, Faculty of Medicine: Alberta Heritage Foundation for Medical Research (AHFMR), HTA.

32. National Health and Medical Research Council (NHMRC), A guide to the development, implementation and evaluation of clinical practice guidelines. 1999, Canberra: NHMRC.

33. Maurer, J., et al., Impact on quality of life after radio(chemo)therapy of head and neck cancer. Strahlenther Onkol, 2011. 187(11): p. 744-749.

34. Zheng, Y., et al., The influence of the 'patient-to-patient model' on swallowing problems in patients with supraglottic laryngeal cancer. Journal of Otorhinolaryngol Related Specialties, 2014. 76(3): p. 171-7. 
35. Argolo, N., et al., Do swallowing exercises improve swallowing dynamic and quality of life in Parkinson's disease? NeuroRehabilitation, 2013. 32(4): p. 949-55.

36. Asadollahpour, F., K. Baghban, and M. Asadi, Validity and Reliability of the Persian Version of the Dysphagia Handicap Index (DHI). Iranian Journal of Otorhinolaryngology, 2015. 27(3): p. 185-191.

37. Bibi, S., et al., The impact of oropharyngeal dysphagia on quality of life in individuals with age over 50 years. Rawal Medical Journal, 2015. 40(1): p. 37-40.

38. Campbell, B.H., et al., Aspiration, weight loss, and quality of life in head and neck cancer survivors. Arch Otolaryngol Head Neck Surg 2004. 130(9): p. 1100-1103.

39. Carlsson, S., et al., Validation of the Swedish M. D. Anderson Dysphagia Inventory (MDADI) in patients with head and neck cancer and neurologic swallowing disturbances. Dysphagia, 2012. 27(3): p. 361-9.

40. da Costa Franceschini, A. and L.F. Mourao, Dysarthria and dysphagia in Amyotrophic Lateral Sclerosis with spinal onset: a study of quality of life related to swallowing. NeuroRehabilitation, 2015. 36(1): p. 127-134.

41. Dos Santos Queija, D., et al., Swallowing and quality of life after total laryngectomy and pharyngolaryngectomy. Braz J Otorhinolaryngol, 2009. 75(4): p. 556-564.

42. Florie, M., et al., Relationship between swallow-specific quality of life and fiber-optic endoscopic evaluation of swallowing findings in patients with head and neck cancer. Head Neck, 2015. 38(1): p. E1848-E1856.

43. Heijnen, B.J., et al., Neuromuscular electrical stimulation versus traditional therapy in patients with parkinson's disease and oropharyngeal dysphagia: Effects on quality of life. Dysphagia, 2012. 27(3): p. 336-345.

44. Lal, P., et al., Is aspiration as detected on pretreatment video fluorography, a harbinger of poor quality of life and early mortality in cancers of the upper aerodigestive tract treated with radiotherapy? South Asian J Cancer, 2014. 3(4): p. 209-212.

45. Lam, P.M. and C.K.Y. Lai, The validation of the chinese version of the Swallow Qualityof-Life Questionnaire (SWAL-QOL) using exploratory and confirmatory factor analysis. Dysphagia, 2011. 26(2): p. 117-124.

46. Leow, L., et al., The impact of dysphagia on quality of life in ageing and Parkinson's disease as measured by the Swallowing Quality of Life (SWAL-QOL) questionnaire. Dysphagia, 2010. 25(3): p. 216-220.

47. Lin, P.H., et al., Effects of functional electrical stimulation on dysphagia caused by radiation therapy in patients with nasopharyngeal carcinoma. Support Care Cancer, 2011. 19(1): p. 91-99.

48. Lovell, S.J., et al., Impact of dysphagia on quality-of-life in nasopharyngeal carcinoma. Head Neck, 2005. 27(10): p. 864-872.

49. McHorney, C.A., et al., The SWAL-QOL and SWAL-CARE outcomes tool for oropharyngeal dysphagia in adults: III: Documentation of reliability and validity. Dysphagia, 2002. 17(2): p. 7-114.

50. Nguyen, N., Frank, C., Moltz, C., Vos, P., Smith, H., Karlsson, U., Dutta, S., Midyett, A., Barloon, J., \& Sallah, S. , Impact of dysphagia on quality of life after treatment of head-and-neck cance. Head and Neck, 2005. 61(3): p. 772-778.

51. Nogueira, D.S., et al., Measuring Outcomes for Dysphagia: Validity and Reliability of the European Portuguese Eating Assessment Tool (P-EAT-10). Dysphagia, 2015. 30(5): p. 511-520. 
52. Pedersen, A., et al., Swallowing outcome measures in head and neck cancer - How do they compare? Oral Oncology, 2016. 52: p. 104-108.

53. Pierce, J.L., et al., Swallowing Disorders in Sjogren's Syndrome: Prevalence, Risk Factors, and Effects on Quality of Life. Dysphagia, 2016. 31(49).

54. Ramaekers, B.L.T., et al., The impact of late treatment-toxicity on generic healthrelated quality of life in head and neck cancer patients after radiotherapy. Oral Oncol, 2011. 47(8): p. 768-774

55. Rogus-Pulia, N., et al., Effects of Device-Facilitated Isometric Progressive Resistance Oropharyngeal Therapy on Swallowing and Health-Related Outcomes in Older Adults with Dysphagia. Journal of the American Geriatric Society, 2016. 64(2): p. 417-24.

56. Starmer, H.M., et al., Patient-perceived long-term communication and swallow function following cerebellopontine angle surgery. Laryngoscope, 2014. 124(2): p. 476-80.

57. Tabor, L., et al., Defining Swallowing-Related Quality of Life Profiles in Individuals with Amyotrophic Lateral Sclerosis. Dysphagia, 2016. 31: p. 376.

58. Vanderwegen, J., G. Van Nuffelen, and M. De Bodt, The validation and psychometric properties of the Dutch version of the Swallowing Quality-of-Life Questionnaire (DSWAL-QOL). Dysphagia, 2013. 28(1): p. 11-23.

59. Zhang, L., et al., Effect of swallowing training on dysphagia and depression in postoperative tongue cancer patients. European Journal of Oncology Nursing, 2014. 18(6): p. 626-629.

60. Zhang, M., et al., Effectiveness of Neuromuscular Electrical Stimulation on Patients With Dysphagia With Medullary Infarction. Arch Phys Med Rehabil, 2016. 97(3): p. 355-362.

61. Chen, A.Y., et al., The development and validation of a dysphagia-specific quality-oflife questionnaire for patients with head and neck cancer: The MD Anderson Dysphagia Inventory. Arch Otolaryngol Head Neck Surg, 2001. 127(7): p. 870-876.

62. Ware, J.E.J., M. Kosinski, and S.D. Keller, SF 36 physical and mental health summary scales: A user's manual. 1994, MA: The Health Institute, New England Medical Center. 
Figure 1 PRISMA flow diagram for reviewing process, adapted from Moher et al. (2009) [30]

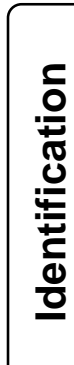

\section{Number of records identified through} PubMed: 1,477
Number of records identified through Embase: 650
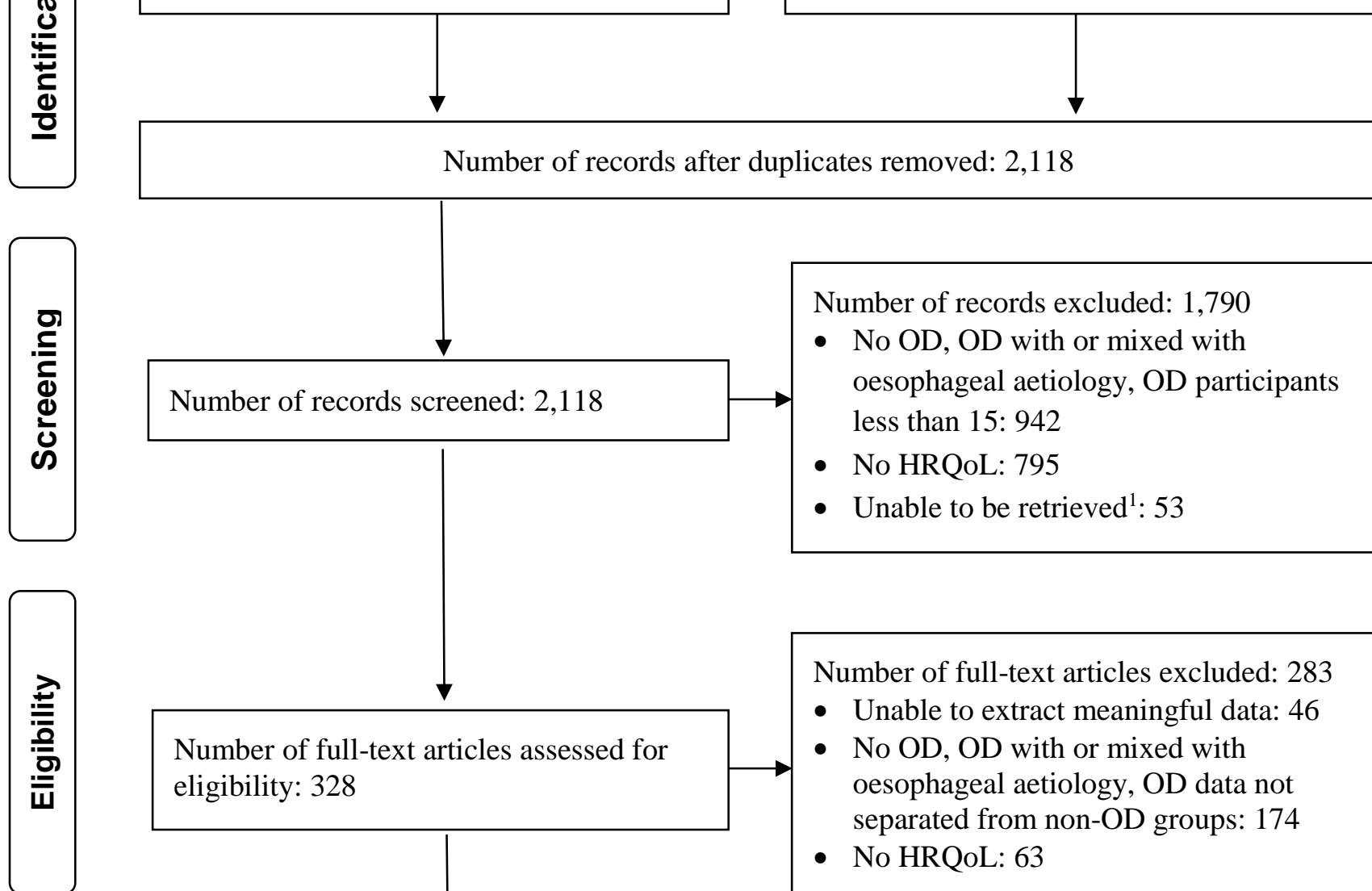

Number of full-text articles excluded: 283

- Unable to extract meaningful data: 46

- No OD, OD with or mixed with

Number of full-text articles assessed for eligibility: 328 oesophageal aetiology, OD data not separated from non-OD groups: 174

- No HRQoL: 63
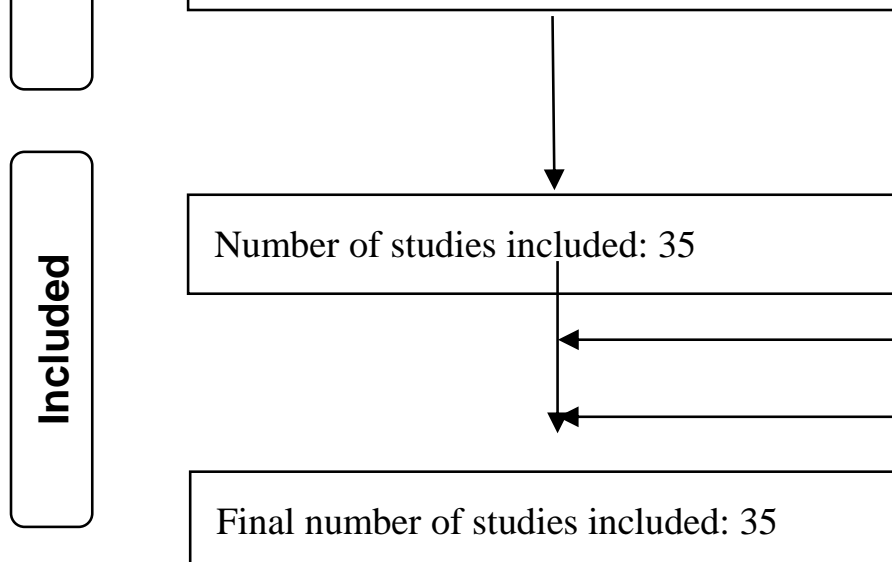

Number of articles added through reference lists: 1

Number of articles excluded after rating methodological quality: 1

${ }^{1}$ Articles not in English, full-text not available or withdrawn (abstracts, posters or conference proceedings) 